\title{
Dynamics of $\mathrm{H}_{2}$ dissociation on the close- packed (111) surface of the noblest metal: $\mathrm{H}_{2}$ $+\mathrm{Au}(111)$
}

Cite as: J. Chem. Phys. 145, 144701 (2016); https://doi.org/10.1063/1.4964486

Submitted: 15 July 2016 . Accepted: 23 September 2016 . Published Online: 11 October 2016

Mark Wijzenbroek, Darcey Helstone, Jörg Meyer (D), and Geert-Jan Kroes (i)

\section{ARTICLES YOU MAY BE INTERESTED IN}

Quantum-state-specific dynamics of the dissociative adsorption and associative desorption of $\mathrm{H}_{2}$ at a $\mathrm{Cu}(111)$ surface

The Journal of Chemical Physics 102, 4625 (1995); https://doi.org/10.1063/1.469511

Dissociation and recombination of $\mathrm{D}_{2}$ on $\mathrm{Cu}(111)$ : $\mathrm{Ab}$ initio molecular dynamics calculations and improved analysis of desorption experiments

The Journal of Chemical Physics 141, 124705 (2014); https://doi.org/10.1063/1.4896058

$\mathrm{Ab}$ initio molecular dynamics calculations on scattering of hyperthermal $\mathrm{H}$ atoms from $\mathrm{Cu}(111)$ and $\mathrm{Au}(111)$

The Journal of Chemical Physics 141, 054705 (2014); https://doi.org/10.1063/1.4891483

The Journal 


\title{
Dynamics of $\mathrm{H}_{2}$ dissociation on the close-packed (111) surface of the noblest metal: $\mathrm{H}_{2}+\mathrm{Au}(111)$
}

\author{
Mark Wijzenbroek, Darcey Helstone, Jörg Meyer, and Geert-Jan Kroes ${ }^{a)}$ \\ Leiden Institute of Chemistry, Gorlaeus Laboratories, Leiden University, P.O. Box 9502, 2300 RA Leiden, \\ The Netherlands
}

(Received 15 July 2016; accepted 23 September 2016; published online 11 October 2016)

\begin{abstract}
We have performed calculations on the dissociative chemisorption of $\mathrm{H}_{2}$ on un-reconstructed and reconstructed $\mathrm{Au}(111)$ with density functional theory, and dynamics calculations on this process on un-reconstructed $\mathrm{Au}(111)$. Due to a very late barrier for dissociation, $\mathrm{H}_{2}+\mathrm{Au}(111)$ is a candidate $\mathrm{H}_{2}$-metal system for which the dissociative chemisorption could be considerably affected by the energy transfer to electron-hole pairs. Minimum barrier geometries and potential energy surfaces were computed for six density functionals. The functionals tested yield minimum barrier heights in the range of 1.15-1.6 eV, and barriers that are even later than found for the similar $\mathrm{H}_{2}+\mathrm{Cu}(111)$ system. The potential energy surfaces have been used in quasi-classical trajectory calculations of the initial $(\mathrm{v}, J)$ state resolved reaction probability for several vibrational states $v$ and rotational states $J$ of $\mathrm{H}_{2}$ and $\mathrm{D}_{2}$. Our calculations may serve as predictions for state-resolved associative desorption experiments, from which initial state-resolved dissociative chemisorption probabilities can be extracted by invoking detailed balance. The vibrational efficacy $\eta_{v=0 \rightarrow 1}$ reported for $\mathrm{D}_{2}$ dissociating on un-reconstructed $\mathrm{Au}(111)$ (about 0.9) is similar to that found in earlier quantum dynamics calculations on $\mathrm{H}_{2}+\mathrm{Ag}(111)$, but larger than found for $\mathrm{D}_{2}+\mathrm{Cu}(111)$. With the two functionals tested most extensively, the reactivity of $\mathrm{H}_{2}$ and $\mathrm{D}_{2}$ exhibits an almost monotonic increase with increasing rotational quantum number $J$. Test calculations suggest that, for chemical accuracy ( $1 \mathrm{kcal} / \mathrm{mol})$, the herringbone reconstruction of $\mathrm{Au}(111)$ should be modeled. Published by AIP Publishing. [http://dx.doi.org/10.1063/1.4964486]
\end{abstract}

\section{INTRODUCTION}

The importance of electronically nonadiabatic effects on reactive scattering and non-reactive scattering of atoms and molecules from metal surfaces is a controversial and hot topic in physical chemistry ${ }^{1-10}$ with relevance to heterogeneous catalysis. ${ }^{11-14}$ In some systems, there is clear evidence that non-adiabatic effects play a dominant role. ${ }^{1,6,10,15-17}$ However, there is also ample evidence that most aspects of the reactive and non-reactive scattering of $\mathrm{H}_{2}$ from metal surfaces under thermal or mild conditions (no photoexcitation of the metal) can be accurately described without taking non-adiabatic effects into account. ${ }^{18}$ Specifically, for $\mathrm{H}_{2}+\mathrm{Pt}(111)$, it was shown that both reaction and diffraction probabilities can be accurately described with a single potential energy surface (PES), which would be unlikely if electron-hole pair (ehp) excitation should be important. ${ }^{2}$ Furthermore, the adiabatic theory is capable of providing a chemically accurate description of energy resolved ${ }^{19}$ as well as initial-state-resolved ${ }^{19,20}$ experiments on reactive and non-reactive ${ }^{19}$ scatterings of $\mathrm{H}_{2}$ from $\mathrm{Cu}(111)$. Likewise, dynamics calculations modeling effects of ehp excitation using the friction theory found little if any effect of ehp excitation processes on the probability of $\mathrm{H}_{2}$ dissociation on metal surfaces. ${ }^{5,21-23}$ This raises the following question: if

\footnotetext{
a) Author to whom correspondence should be addressed. Electronic mail: g.j.kroes@chem.leidenuniv.nl
}

non-adiabatic effects were to be observed on the reactivity of an $\mathrm{H}_{2}$-metal system under thermal or mild conditions, what would be the best system to look at for such effects?

One way to answer the question raised above arises if the assumption made is that non-adiabatic effects on scattering may become important if a (partial) charge transfer from the surface to the molecule occurs during the scattering. ${ }^{24}$ An analysis ${ }^{2}$ of computed ${ }^{25}$ potential energy curves of $\mathrm{H}_{2}$ and $\mathrm{H}_{2}{ }^{-}$in gas phase (see also Ref. 2 for the comparison to the "non-adiabatic" ${ }^{24} \mathrm{NO}$-metal case) then suggests that the most likely candidate $\mathrm{H}_{2}$-metal system for observing non-adiabatic effects should be a system with a very late reaction barrier. Specifically, the computed potential energy curves of $\mathrm{H}_{2}$ and $\mathrm{H}_{2}{ }^{-}$cross at an $\mathrm{H}-\mathrm{H}$ distance of approximately $1.6 \AA$, and the crossing point lies about $3 \mathrm{eV}$ above the minimum of the $\mathrm{H}_{2}$ potential. ${ }^{25}$ In a simple model, the probability that an electron is transferred to the molecule will depend on the sum $\left(\Phi-\mathrm{EA}(\mathrm{r})+\mathrm{V}_{\mathrm{im}}(\mathrm{Z})\right)$ becoming small or negative, ${ }^{24}$ where $\Phi$ is the work function of the metal, EA(r) the electron affinity of the molecule depending on the $\mathrm{H}-\mathrm{H}$ distance $\mathrm{r}$, and $\mathrm{V}_{\mathrm{im}}$ (Z) the image-charge interaction between $\mathrm{H}_{2}{ }^{-}$and the surface, which depends on the molecule-surface distance Z. $\Phi$ does not vary greatly among the late $3 \mathrm{~d}-5 \mathrm{~d}$ transition metal elements belonging to groups 7-11 (from $\mathrm{Fe}$ to $\mathrm{Au}, \Phi$ varies between $4.26 \mathrm{eV}$ for $\mathrm{Ag}$ and $5.65 \mathrm{eV}$ for $\mathrm{Pt}$, the values being $5.1 \mathrm{eV}$ for $\mathrm{Au}$ and $4.65 \mathrm{eV}$ for $\mathrm{Cu}^{26}$ ), and $\mathrm{V}_{\text {im }}(\mathrm{Z})$ does not depend on the metal in the simple model described above. EA(r) starts out at $-3 \mathrm{eV}$ for the gas phase $\mathrm{H}-\mathrm{H}$ distance and rises to $0 \mathrm{eV}$ for 
$\mathrm{r}=1.6 \AA$. Only if the minimum barrier geometry approaches $1.6 \AA$ will there be a reasonably high probability that an electron is transferred to the molecule before the barrier is crossed, thereby allowing the reaction to be affected by this transfer.

The requirement on the barrier geometry discussed above would suggest looking at the interaction of $\mathrm{H}_{2}$ with the "noblest" metals, i.e., $\mathrm{Au}^{27}$ and $\mathrm{Ag}$. Calculations using density functional theory (DFT) put the minimum barrier for $\mathrm{H}_{2}$ dissociation on $\mathrm{Au}(111)$ well above $1 \mathrm{eV},{ }^{27,28}$ and put the barrier position at an $\mathrm{H}-\mathrm{H}$ distance of about $1.2 \AA^{28} .^{28}$ The values are significantly larger than the well established values of the minimum barrier height and position of $\mathrm{H}_{2}+\mathrm{Cu}(111)$ $\left(0.63 \mathrm{eV}\right.$ and $1.03 \AA$, respectively $\left.{ }^{19}\right)$. DFT calculations on the $\mathrm{H}_{2}+\mathrm{Ag}(111)$ system $^{29}$ suggest barrier characteristics $(1.16 \mathrm{eV}, \mathrm{H}-\mathrm{H}$ distance of $1.26 \AA)$ very similar to those of $\mathrm{H}_{2}+\mathrm{Au}(111)$, and $\mathrm{H}_{2}+\mathrm{Ag}(111)$ might therefore also be a good model system for observing non-adiabatic effects on reaction. However, here we focus on $\mathrm{H}_{2}+\mathrm{Au}(111)$.

Although experiments have addressed the importance of ehp excitation on reactive scattering of $\mathrm{H}_{2}$ from Au surfaces, so far the outcome is inconclusive. Experiments on reaction of $\mathrm{H}$ with $\mathrm{H}$ adsorbed to Au observe ehp excitation, which has been attributed to the Langmuir-Hinshelwood recombination reaction. ${ }^{30,31}$ However, by themselves these experiments give no information on the extent to which ehp excitation affects the reverse dissociative chemisorption probability, and ehp excitation is also observed in experiments on reaction of $\mathrm{H}$ with $\mathrm{H}$ adsorbed on $\mathrm{Cu}$ surfaces ${ }^{31}$ although the dissociation of $\mathrm{H}_{2}$ on $\mathrm{Cu}(111)$ is described quite well with electronically adiabatic theory. ${ }^{19,20,32}$ Calculations using ab initio molecular dynamics with electronic friction (AIMDEF) on $\mathrm{H}_{2}+\mathrm{Pd}(100)$ do show that the dissociation of $\mathrm{H}_{2}$ on a metal surface can be accompanied by substantial energy dissipation to ehps, but this dissipation takes place at the product side of the barrier. ${ }^{33}$ In contrast to $\mathrm{H}_{2}, \mathrm{H}$-atoms can get close to metal surfaces, and recent experiments have shown that substantial amounts of translational energy can be dissipated to ehps in $\mathrm{H}$ atoms scattering from $\mathrm{Au}(111) .{ }^{10}$ Therefore, it is likely that the ehp excitation observed in Refs. 30 and 31 takes place at the onset of the associative desorption reaction. Finally, experiments have observed that hot electrons created on Au nanoparticles can promote $\mathrm{H}_{2}$ dissociation, ${ }^{34}$ but these experiments do not involve thermal or mild conditions, as ehp excitations are created by coupling light into plasmons localised on the $\mathrm{Au}$ nanoparticles.

The interaction of $\mathrm{H}_{2}$ with $\mathrm{Au}$ is also of interest in other contexts. Interest in the role gold nanoparticles play in the catalysis of hydrogenation reactions ${ }^{35-38}$ has prompted theoretical studies ${ }^{39-41}$ of interactions of $\mathrm{H}_{2}$ with (defected) $\mathrm{Au}$ clusters and $\mathrm{Au}$ surfaces. Experiments showing effects of the presence of $\mathrm{H}_{2}$ on the conductance through $\mathrm{Au}$ nanowires ${ }^{42}$ have promoted theoretical studies of the dissociative chemisorption of $\mathrm{H}_{2}$ on $\mathrm{Au}$ nanowires. ${ }^{43}$ Experiments ${ }^{44,45}$ and calculations $\mathrm{s}^{45}$ have investigated the effect of alloying $\mathrm{Pd}$ into $\mathrm{Au}$ surfaces on $\mathrm{H}_{2}$ dissociation. Pan et al. have investigated the recombinative desorption of $\mathrm{H}_{2}$ on $\mathrm{Au}(111)$, finding that $\mathrm{H}_{2}$ comes off the surface at a low temperature $(110 \mathrm{~K})$, which is indicative of a weak interaction of atomic $\mathrm{H}$ with $\mathrm{Au}(111) .{ }^{46,47} \mathrm{On} \mathrm{Au}(110)$ $(1 \times 2), \mathrm{H}_{2}$ has been observed to associatively desorb at $216 \mathrm{~K}$, likewise indicating a weak interaction of atomic $\mathrm{H}$ with this surface; these experiments also suggest a very high barrier to dissociative chemisorption of $\mathrm{H}_{2}$ on this $\mathrm{Au}$ surface ${ }^{48}$ Finally, scattering of atomic $\mathrm{H}$ from $\mathrm{Au}(111)$ has been studied theoretically with AIMD, ${ }^{14,49}$ with molecular dynamics (MD) ${ }^{50}$ and with molecular dynamics with electronic friction $(\mathrm{MDEF})^{10}$ calculations, and with experiments. $^{10}$

While this study focuses on $\mathrm{H}_{2}+\mathrm{Au}(111)$, work has also been done on reactive scattering from surfaces of the other coinage metals, $\mathrm{Cu}$ and $\mathrm{Ag}$. We will restrict our overview to the (111) surfaces of these metals. The $\mathrm{H}_{2}+\mathrm{Cu}(111)$ system may be considered a benchmark system, with many experiments and calculations available. Dissociative chemisorption has been studied directly through molecular beam sticking experiments on $\mathrm{H}_{2}{ }^{51,52}$ and $\mathrm{D}_{2},{ }^{53,54}$ and indirectly through associative desorption experiments on $\mathrm{H}_{2}{ }^{52}$ and $\mathrm{D}_{2}{ }^{53}$ and the application of detailed balance. There have also been experiments on rotationally ${ }^{55}$ and vibrationally ${ }^{56-58}$ inelastic scatterings of $\mathrm{H}_{2}$ from $\mathrm{Cu}(111)$. Early high-dimensional quantum dynamics calculations on the reactive scattering include five-dimensional calculations of Gross et al. ${ }^{59}$ and 6D calculations by Dai and Light ${ }^{60,61}$ and Somers et al. ${ }^{62,63}$ Very detailed dynamical studies have been performed using specific reaction parameter functionals, $19,20,23,32,64-67$ also addressing initial-state selected reaction ${ }^{19,20,64}$ as measurable indirectly through associative desorption, as addressed here for $\mathrm{H}_{2}+\mathrm{Au}(111)$.

Much fewer studies have been carried out on $\mathrm{H}_{2}$ $+\operatorname{Ag}(111)$. Experiments on this system have studied dissociative chemisorption indirectly, by looking at associative desorption, ${ }^{68-71}$ while the dissociation has been studied directly with both molecular beam sticking experiments ${ }^{72,73}$ and with six-dimensional quantum dynamics calculations. ${ }^{29}$ The molecular beam experiments were able to measure sticking probabilities up to about 0.02 for average incidence energies up to about $0.48 \mathrm{eV}$. Higher incidence energies (up to about $0.8 \mathrm{eV}$ ) can be achieved by using $\mathrm{H}_{2}$ as seeding gas, but the experimentalists reported that with the detection technique that needs to be applied in these experiments (the King and Wells technique ${ }^{74}$ ), the reaction could not be detected (this would have required sticking probabilities $\geq 0.05) .{ }^{72}$ Similar difficulties should be expected for $\mathrm{H}_{2}+\mathrm{Au}(111)$, which exhibits similarly high reaction barriers as $\mathrm{H}_{2}+\mathrm{Ag}(111)$ (see below). For this reason, in the present paper on $\mathrm{H}_{2}+\mathrm{Au}(111)$, we focus on making predictions for associative desorption experiments, which have the added advantage of producing rovibrational state-selected results that are better resolved with respect to translational energy.

The goal of our work is to provide predictions of initial state-selected reaction probabilities, which can be tested through experiments that look at dissociative chemisorption indirectly, by measuring associative desorption of $\mathrm{H}_{2}\left(\right.$ or $\left.\mathrm{D}_{2}\right)$ in a state-selective manner and applying detailed balance. ${ }^{52,53}$ As detailed below, we perform dynamics calculations using PESs based on six different functionals, among which are the well-known $\mathrm{PBE}^{75}$ and $\mathrm{RPBE}^{76}$ functionals, and 
the SRP48 $8^{32}$ functional that was shown to work well for $\mathrm{H}_{2}+\mathrm{Cu}(111)^{19}$ and might for this reason be expected to also yield a reasonable description of $\mathrm{H}_{2}+\mathrm{Au}(111)$. Subsequent experimental measurements might show whether any of the predicted set of reaction probabilities, which are all obtained here within the Born-Oppenheimer approximation, do reasonably well at predicting the outcome of experiments for a wide range of rotational and vibrational states. Large deviations from the theoretical predictions might serve as an indication that the ehp excitation could be important for the $\mathrm{H}_{2}+\mathrm{Au}(111)$ system.

This paper is organized as follows. Section II A describes the dynamical model, Section II B the construction of PESs, Section II C the dynamics methods used to study $\mathrm{H}_{2}+\mathrm{Au}(111)$, and Section II D provides computational details. In Section III A, we briefly discuss the results of the electronic structure calculations, while Section III B reports our predictions for the calculated initial-state selected reaction probabilities. Section III C describes how reaction probabilities can be fitted to reaction probability curves, to facilitate their use in the prediction of time-of-flight spectra for comparison to actual state-resolved associative desorption experiments. Conclusions are provided in Section IV.

\section{METHOD}

\section{A. Dynamical model}

The calculations use the Born-Oppenheimer static surface (BOSS) approximation and most of our calculations model the $\mathrm{Au}(111)$ surface as un-reconstructed. That is, we make the Born-Oppenheimer approximation and assume the reaction takes place on the ground state PES and we assume the surface atoms to be static and to occupy their ideal, relaxed $0 \mathrm{~K}$ lattice configuration positions in the un-reconstructed (111) surface of the fcc metal gold. Although we realize that $\mathrm{Au}(111)$ reconstructs to a surface with a herringbone pattern, ${ }^{77,78}$ like in most computational studies this reconstruction is not taken into account in most of our calculations. Doing so would at least require the use of a very large $(22 \times \sqrt{3})$ surface unit cell, and even then the domain boundaries between different orientations of the reconstruction, which are found at finite temperatures, would not be taken into account. ${ }^{79}$

As a result of the chosen dynamical model, only the motion in the six molecular degrees of freedom of $\mathrm{H}_{2}$ is taken into account. In Figure 1(a) we show the coordinate system used for our study, and Figure 1(b) shows the surface unit cell for the un-reconstructed $\mathrm{Au}(111)$ surface and its positioning relative to the coordinates used for $\mathrm{H}_{2}$.

\section{B. Construction of potential energy surfaces}

In the first step of computing observables within a Born-Oppenheimer approach, six functionals were used to solve the electronic Schrödinger equation with DFT for several configurations of the system, in order to construct full six-dimensional (6D) PESs. Three of the functionals chosen use PBE correlation, i.e., the PBE ${ }^{75}$ the RPBE ${ }^{76}$ (a)

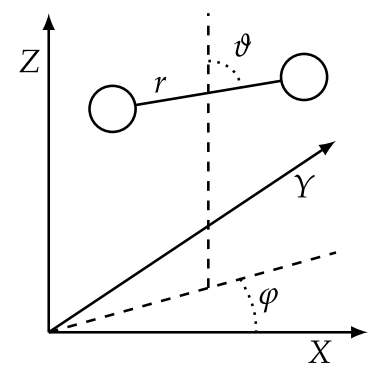

(b)

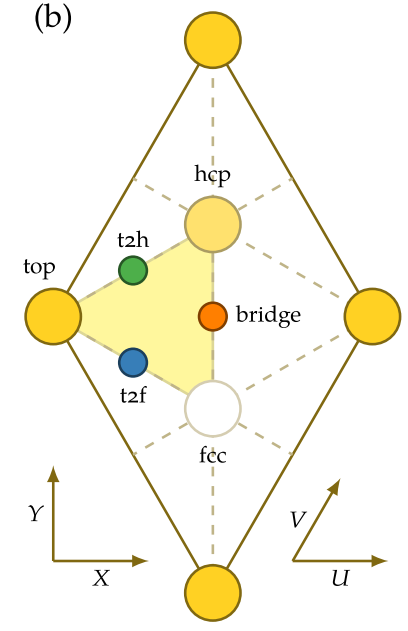

FIG. 1. (a) The center of mass coordinate system used for the description of the $\mathrm{H}_{2}$ molecule relative to un-reconstructed $\mathrm{Au}(111)$. (b) The surface unit cell and the sites considered for the un-reconstructed $\mathrm{Au}(111)$ surface, and the relationship with the coordinate system chosen for $\mathrm{H}_{2}$ relative to $\mathrm{Au}(111)$. The origin $(X, Y, Z)=(0,0,0)$ of the center of mass coordinates is located in the surface plane at a top site. Polar and azimuthal angles, $\theta$ and $\phi$, are chosen such that $\left(\theta=90^{\circ}, \phi=0^{\circ}\right)$ corresponds to molecules parallel to the surface along the $X$ (or equivalently $U$ ) direction.

and the SRP48 ${ }^{32}$ functional, with the SRP48 functional being a weighted average of the first two functionals $(0.48 * \mathrm{RPBE}+0.52 * \mathrm{PBE})$. The latter functional allows molecular beam sticking and associative desorption experiments on $\mathrm{D}_{2}+\mathrm{Cu}(111)^{53}$ to be reproduced with chemical accuracy, ${ }^{20,32}$ and was based on an earlier version ${ }^{19}$ also correctly describing molecular beam sticking and associative desorption experiments ${ }^{52}$ and rotationally inelastic scattering experiments ${ }^{55}$ on $\mathrm{H}_{2}+\mathrm{Cu}(111)$. The three other functionals chosen employ the vdW-DF1 (henceforth simply called vdWDF) correlation functional developed by the Chalmers-Rutgers group. ${ }^{80}$ Three functionals are obtained by combining this correlation functional with $\mathrm{PBE}^{75}$ (PBE-vdW-DF), RPBE, ${ }^{76}$ and optPBE ${ }^{81}$ exchange. Of these, the last is of special interest because the optPBE-vdW-DF functional shows chemical accuracy for the S22 database of van der Waals molecules, ${ }^{81}$ and because in a study investigating $4 \mathrm{H}_{2}$-metal surface systems it gave a slightly better overall description of molecular beam sticking experiments on $\mathrm{H}_{2}+$ metal systems than SRP48. ${ }^{82}$

To arrive at global expressions for the PES, DFT data were computed on grids of points and interpolated with the accurate corrugation reducing procedure (CRP) ${ }^{83,84}$ The procedure followed is analogous to that used earlier for $\mathrm{H}_{2}+\mathrm{Ru}(0001),{ }^{85}$ with the only difference being that the switch to the gas phase $\mathrm{H}_{2}$ potential is now only complete at a molecule-surface distance of $6.5 \AA$ (see Ref. 85 for details). We used the $\mathrm{p} 3 \mathrm{~m} 1$ plane group symmetry ${ }^{86}$ associated with the $\mathrm{Cu}(111)$ surface. For details, the reader is referred to Ref. 85 .

\section{Dynamics method}

In the second step of the Born-Oppenheimer approach selected, dynamical observables are computed with the quasiclassical trajectory (QCT) method, ${ }^{87}$ i.e., with initial energy 
put into vibration taking into account the zero-point energy. The QCT method has been shown to be remarkably accurate for dissociative chemisorption of $\mathrm{D}_{2}$ and even $\mathrm{H}_{2}$ for a range of systems, including $\mathrm{H}_{2}+\mathrm{Cu}(111),{ }^{19,64,67} \mathrm{Cu}(100),{ }^{88}$ $\mathrm{Ru}(0001),{ }^{85}$ and $\operatorname{Pt}(111) .{ }^{89}$

Observables are computed by running trajectories for an ensemble of initial conditions. The molecules are initially put $7 \AA$ away from the surface, and given a velocity normal towards the surface that corresponds to the incidence energy selected. The impact site on the surface is chosen at random. The orientation of the molecule, $\theta$ and $\phi$ (Fig. 1(a)), is randomly chosen based on the selection of the rotational state: the magnitude of the classical initial angular momentum $L$ is fixed by $L=\sqrt{J(J+1)} / \hbar$, and its orientation is taken randomly but with the constraint that $\cos \vartheta_{L}=m_{J} / \sqrt{J(J+1)}$, where $J$ is the rotational quantum number, $m_{J}$ is the magnetic rotational quantum number (the surface normal being the projection axis), and $\vartheta_{L}$ the angle between the angular momentum vector and the surface normal. To take into account the initial vibrational energy of the molecule, the vibrational states of $\mathrm{H}_{2}$ are computed using the Fourier grid Hamiltonian method. ${ }^{90}$ The molecule is given the amount of energy corresponding to a specific vibrational level by randomly sampling positions and momenta from a one-dimensional quasi-classical dynamics calculation of vibrating $\mathrm{H}_{2}$ for the corresponding energy.

In the trajectories, the method of Stoer and Burlisch ${ }^{91}$ is used to propagate the equations of motion. In the calculation of reaction probabilities, in a trajectory a molecule is considered dissociated if its $\mathrm{H}_{2}$ distance becomes greater than $2.5 \AA$. The reaction probability is computed from $P_{r}=N_{r} / N_{\text {total }}$, where $N_{r}$ is the number of reactive trajectories and $N_{\text {total }}$ is the total number of trajectories run for a specific incidence condition (typically taken equal to $10^{4}$ ). For a given initial vibrational state $v$ and rotational state $J$, the degeneracy averaged reaction probability $P_{r}(v, J)$ is calculated as

$$
P_{r}(v, J)=\sum_{m_{J}=0}^{J}\left(2-\delta_{m_{J} 0}\right) P_{r}\left(v, J, m_{J}\right) /(2 J+1),
$$

where $P_{r}\left(v, J, m_{J}\right)$ is a fully initial state resolved reaction probability.

Other quantities of interest are the vibrational efficacies $\eta_{v=0 \rightarrow 1}$ and $\eta_{v=1 \rightarrow 2}$, and the rotational efficacy $\eta_{r o t}$. The former describe how efficient putting energy into vibration prior to the collision is at promoting reaction relative to putting energy in translation, while the latter describes how efficiently rotational pre-excitation promotes the reaction. These are typically computed for a particular value of the reaction probability $R$ as

$\eta_{v=v_{a} \rightarrow v_{b}}(R)=\frac{E_{i}\left[P_{r}\left(v_{a}, J_{c}\right)=R\right]-E_{i}\left[P_{r}\left(v_{b}, J_{c}\right)=R\right]}{E\left(v=v_{b}, J=J_{c}\right)-E\left(v=v_{a}, J=J_{c}\right)}$.

In Eq. (2), $E_{i}\left[P_{r}(v, J)=R\right]$ is the incidence energy at which the initial state-resolved reaction probability first becomes equal to $R$, for $\mathrm{H}_{2}$ initially in its $(v, J)$ state. Furthermore, $E\left(v=v_{i}, J=J_{c}\right)$ is the internal energy of $\mathrm{H}_{2}$ in its initial $\left(v_{i}, J_{c}\right)$ rovibrational state. In this work, we choose $J_{c}=3$ for $\mathrm{H}_{2}$ (odd $J$-states being more abundant for $\mathrm{H}_{2}$ ) and $J_{c}=2$ for
$\mathrm{D}_{2}$ (even $J$-states being more abundant for $\mathrm{D}_{2}$ ). The rotational efficacy is evaluated as

$\eta_{r o t}(R)=\frac{E_{i}\left[P_{r}(0, J=8)=R\right]-E_{i}\left[P_{r}(0, J=10)=R\right]}{E(v=0, J=10)-E(v=0, J=8)}$.

The rovibrational energies were computed using the Fourier grid Hamiltonian method ${ }^{90}$ on the basis of our DFT calculations. In this work, we usually choose $R=0.25$, which is equal to approximately half the maximum reaction probability (or saturation value of the reaction probability) that could be fitted earlier to associative desorption and molecular beam experiments on $\mathrm{D}_{2}+\mathrm{Cu}(111){ }^{20}$

\section{Computational details}

The electronic structure calculations on $\mathrm{H}_{2}$ interacting with un-reconstructed $\mathrm{Au}(111)$ were done with version 5.2.12 of the VASP software package. ${ }^{92,93}$ The calculations employing the PBE correlation functional used the standard ${ }^{94}$ VASP ultrasoft pseudopotentials ${ }^{95}$ while the calculations employing the vdW-DF correlation functional used the standard ${ }^{93}$ VASP projector augmented wave (PAW) ${ }^{96}$ potentials. VASP allows the efficient evaluation of the nonlocal vdW-DF correlation functional with a scheme due to Román-Pérez and Soler. ${ }^{97}$

For each functional, the bulk fcc lattice constant was computed using a $20 \times 20 \times 20$ grid of k-points and a planewave cutoff energy of $500 \mathrm{eV}$. Lattice constants computed were $4.1967 \AA$ for the optPBE-vdW-DF and $4.2022 \AA$ for SRP48 functional, respectively. Compared to the experimental value $\left(4.08 \AA^{98}\right)$, these functionals overestimate the lattice constant by about $3 \%$. Lattice constants computed for the other functionals may be found in Table S1 of the supplementary material.

Slabs were generated by carrying out a relaxation of the interlayer distances of a four-layer slab using a $20 \times 20 \times 1$ grid of k-points and again a plane-wave cutoff energy of $500 \mathrm{eV}$. The calculations of the PESs for $\mathrm{H}_{2}+\mathrm{Au}(111)$ used static four-layer slabs with the interlayer distances fixed to the values found through these relaxation calculations. The calculations employed a $2 \times 2$ surface unit cell, a plane wave energy cutoff of $400 \mathrm{eV}$, and $11 \times 11 \times 1 \mathrm{k}$-points. There is a $13 \AA$ vacuum between the periodic images of the slabs, and Fermi-smearing with a width of $0.1 \mathrm{eV}$ was used. With the parameters used, and within the limits of the frozen-core potentials, the estimated convergence of the DFT calculations was $30 \mathrm{meV}$. As an example of a convergence test, in Table I we present results on the convergence of the molecule-surface interaction energy with respect to the number of layers $n_{L}$ in the slab. For $n_{L} \geq 5$ the interaction energies show small odd-even oscillations, which we have also observed for other systems. The results show very good convergence if averages are taken over the results for $n_{L}$ and $n_{L}+1$ with $n_{L} \geq 5$ and equal to an odd number, and the results for $n_{L}=4$ (as used in our PES calculations for computational efficiency) are in good agreement with these averages.

We have also carried out a few calculations using PBEvdW-DF for $\mathrm{H}_{2}$ adsorption on herringbone-reconstructed $\mathrm{Au}(111)$, to examine how the reconstruction might affect the dissociation barrier. Here we employ the relaxed geometry 
TABLE I. Convergence tests on the dependence of the interaction energy of $\mathrm{H}_{2}$ with $\mathrm{Au}(111)$ on the number of layers $n_{L}$ in the Au slab, for two fixed geometries of the molecule with respect to the surface, corresponding to the top-to-bridge (ttb, inset Fig. 2(a)) and bridge-to-hollow (bth, Fig. 2(b), inset, but with $\mathrm{H}_{2}$ rotated by $90^{\circ}$ in $\phi$ so that dissociation occurs to one fcc hollow and one hcp hollow site) geometries. The calculations used the PBE functional, a plane-wave cutoff of $400 \mathrm{eV}$, and $9 \times 9 \times 1 \mathrm{k}$-points.

\begin{tabular}{lcc}
\hline \hline$n_{L}$ & $E_{t t b}(\mathrm{eV})$ & $E_{b t h}(\mathrm{eV})$ \\
\hline 3 & 1.252 & 1.181 \\
4 & 1.229 & 1.247 \\
5 & 1.224 & 1.228 \\
6 & 1.252 & 1.291 \\
7 & 1.204 & 1.226 \\
8 & 1.261 & 1.304 \\
$(5+6) / 2$ & 1.238 & 1.260 \\
$(7+8) / 2$ & 1.233 & 1.265 \\
\hline \hline
\end{tabular}

for the $(22 \times \sqrt{3})$ surface unit cell based on PBE-vdWDF as published by Hanke and Björk (HB) as part of the supplementary material of Ref. 79. The slab consists of six layers in order to accurately capture the delicate rumpling of the top four surface layers, while in the bottom two layers the atoms have been kept frozen at their ideal bulk positions. Following HB, we have used a $1 \times 8 \times 1$ Monkhorst-Pack grid for the Brillouin zone integration. ${ }^{79}$ Remaining settings have been chosen consistently with the computational setup for PBE-vdW-DF calculations of the un-reconstructed Au(111) surface as detailed above. Within our computational setup, we could then reproduce the adsorption energies for $\mathrm{H}$-atoms published by HB (Figure 4, top panel of Ref. 79) to within $10 \mathrm{meV}$.

\section{RESULTS AND DISCUSSION}

\section{A. Electronic structure calculations and potential energy surfaces}

Figure 2 shows elbow plots of the PES computed with the SRP48 functional for four configurations in which $\mathrm{H}_{2}$ is parallel to the model $\mathrm{Au}(111)$ surface, for impact on the high symmetry top, bridge, hcp hollow, and for one additional configuration in which $\mathrm{H}_{2}$ impacts on a site ( $2 \mathrm{~h}$ ) midway between a top and hcp site, respectively (see also Figure 1(b)). Table II lists the geometries and heights of the barrier to dissociation found for the corresponding and two additional geometries, also providing data for the optPBE-vdW-DF functional. The analogous results for the other functionals are in Tables S2 and S3 of the supplementary material.

Our calculations with the SRP48 density functional put the $\mathrm{H}-\mathrm{H}$ distance at the barrier $\left(r_{b}\right)$ at values in the range $1.35-1.52 \AA$ for the configurations considered in Figure 2 and Table II (not counting the most repulsive t $2 \mathrm{~h}, \phi=30^{\circ}$ configuration, and the bridge-to-hollow configuration here and in the subsequent analysis, the bridge-to-hollow configuration is the configuration with the center-of-mass of $\mathrm{H}_{2}$ located as in the inset to Fig. 2(b), but with the molecule rotated by $\phi=90^{\circ}$ so that the atoms dissociate to the fcc and hcp hollow sites). The optPBE-vdW-DF functional yields a range of somewhat smaller values (1.31-1.47 $\AA$ ). Nevertheless, for both functionals, these values come close to the value (1.6 $\mathrm{A})$ at which the $\mathrm{H}_{2}$ and $\mathrm{H}_{2}{ }^{-}$curves cross in vacuum, ${ }^{25}$ suggesting that $\mathrm{H}_{2}+\mathrm{Au}(111)$ might be a candidate for a system affected by ehp excitation, as discussed in the Introduction. The $r_{b}$ values obtained with the PBE and RPBE functionals (Table S2 of the supplementary material) do not differ much from the values calculated with SRP48 (Table II), and the PBE-vdW-DF

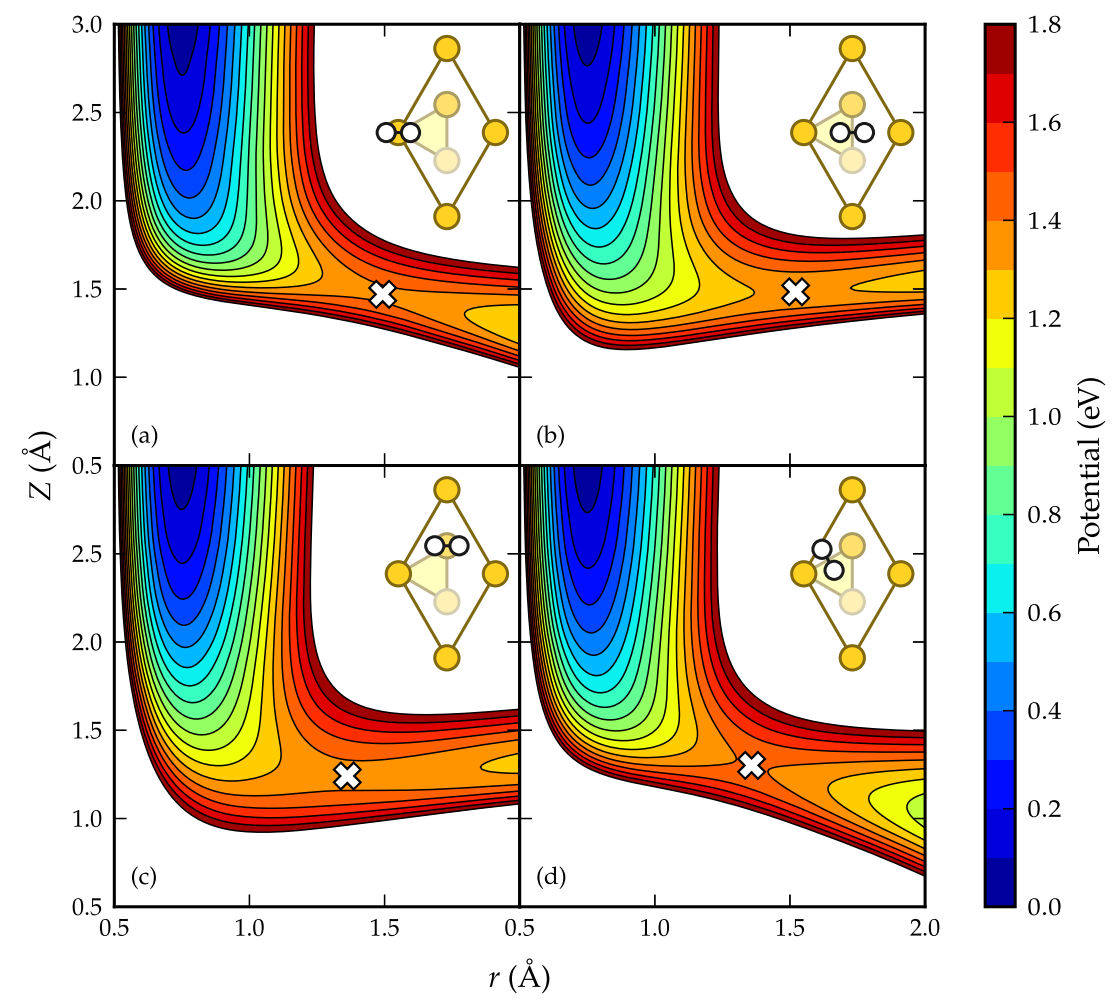

FIG. 2. Elbow plots (i.e., $V(Z, r)$ ) resulting from the $\mathrm{H}_{2}+\mathrm{Au}(111)$ PES computed with the SRP48 functional and interpolated with the CRP method for four high symmetry configurations with the molecular axis parallel to the surface $\left(\theta=90^{\circ}\right)$ as depicted by the insets, for (a) the top site and $\phi=0^{\circ}$, (b) the bridge site and $\phi=0^{\circ}$ (the bridge-totop global minimum barrier geometry), (c) the hcp site and $\phi=0^{\circ}$, and (d) the t2h site and $\phi=120^{\circ}$. Barrier geometries are indicated with white crosses, and the corresponding barrier heights are given in Table II. 
TABLE II. The $\mathrm{H}-\mathrm{H}$ distance $r_{b}$ and the $\mathrm{H}_{2}$-surface distance $Z_{b}$ at the minimum barrier geometry, and the minimum barrier height $E_{b}$ are provided for configurations in which $\mathrm{H}_{2}$ is parallel to the $\mathrm{Au}(111)$ surface $\left(\theta=90^{\circ}\right)$. Results are provided for the SRP48 and for the optPBE-vdW-DF functional.

\begin{tabular}{lccccccc}
\hline \hline & \multicolumn{3}{c}{ SRP 48 } & & \multicolumn{3}{c}{ optPBE-vdW-DF } \\
\cline { 2 - 4 } Configuration & $\begin{array}{c}r_{b} \\
(\AA)\end{array}$ & $\begin{array}{c}Z_{b} \\
(\AA)\end{array}$ & $\begin{array}{c}E_{b} \\
(\mathrm{eV})\end{array}$ & & $\begin{array}{c}r_{b} \\
(\AA)\end{array}$ & $\begin{array}{c}Z_{b} \\
(\AA)\end{array}$ & $\begin{array}{c}E_{b} \\
(\mathrm{eV})\end{array}$ \\
\hline Top, $\phi=0^{\circ}$ & 1.493 & 1.470 & 1.382 & & 1.473 & 1.483 & 1.379 \\
Bridge, $\phi=0^{\circ}$ & 1.521 & 1.484 & 1.315 & & 1.420 & 1.486 & 1.288 \\
Bridge, $\phi=90^{\circ}$ & 1.180 & 1.089 & 1.407 & & 1.200 & 1.098 & 1.508 \\
hcp, $\phi=0^{\circ}$ & 1.362 & 1.241 & 1.370 & & 1.307 & 1.262 & 1.407 \\
t2h, $\phi=120^{\circ}$ & 1.358 & 1.301 & 1.407 & & 1.360 & 1.312 & 1.445 \\
t2h, $\phi=30^{\circ}$ & 1.689 & 1.552 & 1.783 & & 1.652 & 1.565 & 1.761 \\
\hline \hline
\end{tabular}

and RPBE-vdW-DF values (Table S3 of the supplementary material) do not differ much from the optPBE-vdW-DF values (Table II).

To test whether the $\mathrm{H}_{2}$ molecule can pick up charge from the surface at the transition state geometry for $\mathrm{H}_{2}+\mathrm{Au}(111)$, we performed a Bader charge analysis ${ }^{99-103}$ of the optPBEvdW-DF electron densities. The results for the bridge-totop barrier geometry (Table III) indicate a negligible charge transfer from the surface to the molecule (the result in Table III might be taken to indicate charge transfer from the molecule to the surface, but within the accuracy of the analysis method the result is consistent with no charge transfer). This result is at odds with the results obtained with the SRP48 functional ${ }^{32}$ for the bridge-to-hollow geometry for $\mathrm{H}_{2}+\mathrm{Cu}(111)$, which indicates a charge transfer from the surface to the molecule of 0.23 . This partial charge transfer did not preclude a chemically accurate description of $\mathrm{H}_{2}+\mathrm{Cu}(111),{ }^{19,32}$ although a more massive charge transfer has been suggested to lead to a breakdown of DFT within the generalized gradient approximation (GGA) in the work on $\mathrm{O}_{2}+\mathrm{Al}(111) .{ }^{104}$ The observation of no charge transfer from the surface to the molecule in the $\mathrm{H}_{2}+\mathrm{Au}(111)$ transition state could be taken to suggest that there should be no problem with a description of the system at the DFT/GGA level of theory, and that electron transfer from the surface to the molecule and back should not be able to drive electron-hole pair excitation as it does for the highly vibrationally excited NO scattering from metal surfaces. ${ }^{1,24}$ We have also tested whether the difference in charge transfer at the minimum barrier geometry between $\mathrm{H}_{2}+\mathrm{Au}(111)$ and $\mathrm{H}_{2}+\mathrm{Cu}(111)$ could be due to the differences between the geometries
TABLE IV. The H-H distance $r_{b}$ and the minimum barrier height $E_{b}$ at the bridge-to-hollow barrier geometry obtained with the PBE functional (for Ag and $\mathrm{Au}$ ) and the PW91 functional (for $\mathrm{Cu}$ ) are provided for $\mathrm{H}_{2}+\mathrm{Cu}(111)$, $\mathrm{Ag}(111)$, and $\mathrm{Au}(111)$. In all cases, $\mathrm{H}_{2}$ is parallel to the surface $\left(\theta=90^{\circ}\right)$.

\begin{tabular}{lccc}
\hline \hline System & $r_{b}(\AA)$ & $E_{b}(\mathrm{eV})$ & Reference \\
\hline $\mathrm{H}_{2}+\mathrm{Cu}(111)$ & 1.01 & 0.49 & 19 \\
$\mathrm{H}_{2}+\mathrm{Ag}(111)$ & 1.26 & 1.16 & 29 \\
$\mathrm{H}_{2}+\mathrm{Au}(111)$ & 1.19 & 1.25 & This work \\
$\mathrm{H}_{2}+\mathrm{Au}(111)$ & 1.2 & 1.35 & 28 \\
\hline \hline
\end{tabular}

(bridge-to-top for $\mathrm{Au}$ and bridge-to-hollow for $\mathrm{Cu}$ ), but additional calculations for the bridge-to-hollow minimum barrier geometry of $\mathrm{H}_{2}+\mathrm{Au}(111)$ suggest that this is not the case (see Table III).

We may also compare the $r_{b}$ and $E_{b}$ values calculated here with $\mathrm{PBE}$ for $\mathrm{H}_{2}+\mathrm{Au}(111)$ to those of $\mathrm{H}_{2}+\mathrm{Cu}(111)$ and $\mathrm{Ag}(111)$. For the global minimum barrier geometry obtained for $\mathrm{H}_{2}$ on $\mathrm{Cu}(111)$ (the bridge-to-hollow configuration), the corresponding values are given in Table IV for all three surfaces. The comparison shows that the barrier for $\mathrm{H}_{2}$ dissociation on $\mathrm{Au}(111)$ is of similar height as that for $\mathrm{H}_{2}$ dissociation on $\mathrm{Ag}(111)$, but much higher than on $\mathrm{Cu}(111)$. For this geometry, we also predict the barrier for $\mathrm{H}_{2}$ on $\mathrm{Au}(111)$ to be later (occurring at a larger value of $r_{b}$, i.e., $1.2 \AA$ ) than on $\mathrm{Cu}(111)(1.0 \AA)$. We note that the orientation of the molecule differs from that in the actual minimum barrier geometry on the bridge site of $\mathrm{Au}(111)$, where the minimum barrier is found for bridge-to-top dissociation (see also Table II). For this geometry, we predict the barrier for $\mathrm{H}_{2}$ on $\mathrm{Au}(111)$ to be even later (at $r_{b} \approx 1.5 \AA$, see Table $\mathrm{S} 2$ of the supplementary material). Application of Polanyi's rules, ${ }^{105}$ and the late minimum barriers found for all impact sites, then suggests that it should be much easier to promote dissociation of $\mathrm{H}_{2}$ on $\mathrm{Au}(111)$ by pre-exciting the $\mathrm{H}_{2}$ vibration than on $\mathrm{Cu}(111)$, on which pre-exciting the vibration is about a factor 0.5-0.6 as effective as promoting the reaction by enhancing the incident translational energy. ${ }^{52}$

The $r_{b}$ value calculated here for bridge-to-hollow dissociation with PBE for $\mathrm{H}_{2}+\mathrm{Au}(111)(1.19 \AA$, see Table IV) compares well with the PBE value of Libisch et al. $^{28}(1.2 \AA$, see also Table IV), but there is a fairly sizeable difference between the barrier heights (we compute a PBE value of $1.25 \mathrm{eV}$, Libisch et al. obtain $1.35 \mathrm{eV},{ }^{28}$ see Table IV). The difference in barrier height could be due to several differences between the DFT methodologies used in the two sets of calculations.

TABLE III. Excess charge in units of $\mathrm{e}^{-}$transferred from the metal surface to the dissociating molecules for the transition states of $\mathrm{H}_{2}+\mathrm{Au}(111)$ and $\mathrm{H}_{2}+\mathrm{Cu}(111)$, and for the bridge-to-hollow minimum barrier geometry of $\mathrm{H}_{2}+\mathrm{Au}(111)$. The values are calculated as the difference between the charge of the molecule in the gas phase and the charge of the molecule at the transition state (or other minimum barrier geometry), using a Bader charge analysis.

\begin{tabular}{llcccc}
\hline \hline System & Configuration & $r_{b}(\AA)$ & $Z_{b}(\AA)$ & Excess charge $\left(\mathrm{e}^{-}\right)$ & Functional \\
\hline $\mathrm{H}_{2}+\mathrm{Au}(111)$ & Bridge-to-top & 1.42 & 1.49 & -0.02 & optPBE-vdW-DF \\
$\mathrm{H}_{2}+\mathrm{Au}(111)$ & Bridge-to-hollow & 1.20 & 1.10 & -0.02 & optPBE-vdW-DF \\
$\mathrm{H}_{2}+\mathrm{Cu}(111)$ & Bridge-to-hollow & 1.03 & 1.16 & 0.23 & SRP48 \\
\hline \hline
\end{tabular}


The calculations of Libisch et al. used better pseudo-potentials (PAW) than we used with PBE (ultrasoft pseudo-potentials), a larger supercell $(3 \times 3$ instead of $2 \times 2)$, and a thicker slab (7 layers instead of four), and they minimized artificial electrostatic interactions by adsorbing $\mathrm{H}_{2}$ on both sides of the slab. At the same time, they used a smaller plane-wave cutoff energy $(250 \mathrm{eV})$ than we did $(400 \mathrm{eV})$, and reported convergence problems with their spin-polarized calculations that we did not observe with our spin-unpolarized setup. We do not know the reason for the $0.1 \mathrm{eV}$ difference between our results and those of Libisch et al.; the discrepancy cannot be explained from the difference in the number of layers used in the calculations (see Table I and its discussion in Section II D).

Features that are important determinants of the appearance of the reaction probability curve are the minimum barrier height and the energetic corrugation, ${ }^{85,106}$ which are shown for the six functionals used in Figure 3. Here, the energetic corrugation $\Xi$ is defined as the difference between the barrier height for the most repulsive high symmetry configuration (found to be $\mathrm{t} 2 \mathrm{~h}, \phi=30^{\circ}$ ) and the configuration with the lowest barrier height (bridge-to-top). As Figure 3 shows, the six functionals used differ little in the value of $\Xi$ obtained with them (in the range $0.46-0.51 \mathrm{eV}$ ), but they differ greatly in the minimum barrier height (in the range $1.17 \mathrm{eV}$ for PBE to $1.57 \mathrm{eV}$ for RPBE-vdW-DF). As the value of $\Xi$ mostly determines the slope of the reaction probability curve (which is inversely related to $\Xi$ ) and the $\Xi$ values are all rather similar, one would expect the reaction probability curves computed with different functionals to be rather similar in shape, but displaced from one another along the energy axis with offsets determined by the differences in the computed minimum barrier heights.

Finally, we have also examined the effect the herringbone reconstruction of $\mathrm{Au}(111)$ might have on the minimum barrier height, which has been consistently obtained at a bridge-totop like configuration (with angles $\theta=90^{\circ}, \phi=0^{\circ}$, see Fig. 1 and inset to Fig. 2(b)) by all functionals employed in this

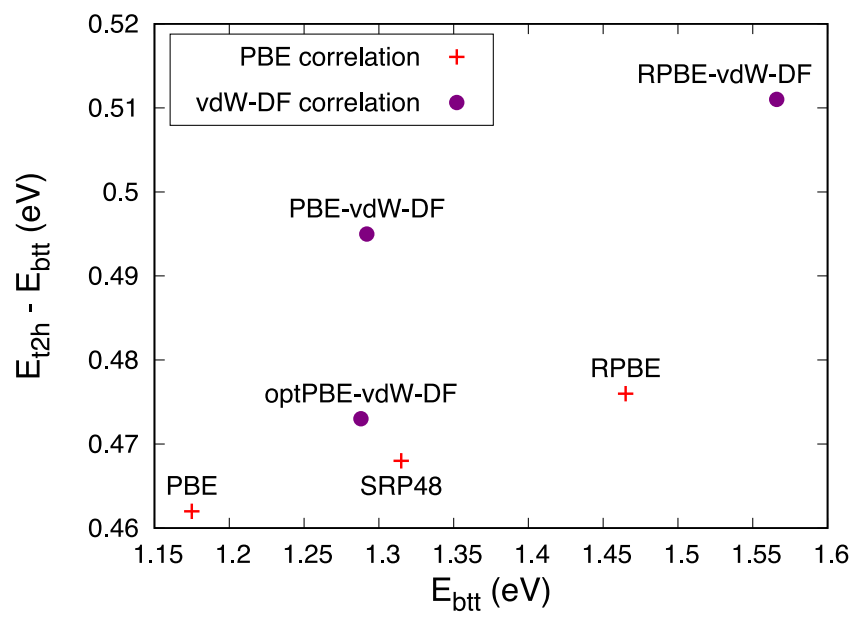

FIG. 3. The energetic corrugation (see text for definition) versus the minimum barrier height for $\mathrm{H}_{2}$ interacting with un-reconstructed $\mathrm{Au}(111)$ is shown for the six density functionals used. Results obtained with functionals employing PBE correlation are marked with red symbols, and results obtained with vdW-DF correlation with purple symbols.
TABLE V. Barrier heights $E_{b}$ based on PBE-vdW-DF obtained in different regions of the herringbone reconstruction of $\mathrm{Au}(111)$ as described by a $(22 \times \sqrt{3})$ surface unit cell obtained by Hanke and Björk. ${ }^{79}$ In all cases $\mathrm{H}_{2}$ is placed at the global minimum barrier geometry obtained at the bridge-to-top configuration $\left(\theta=90^{\circ}, \phi=0^{\circ}\right)$. The difference in energy to this configuration on the un-reconstructed $\mathrm{Au}(111)$ surface is given by $\Delta E_{b}$.

\begin{tabular}{lcc}
\hline \hline PBE-vdW-DF & $E_{b}(\mathrm{eV})$ & $\Delta \mathrm{E}_{b}(\mathrm{meV})$ \\
\hline Un-reconstructed $\mathrm{Au}(111)$, bridge-to-top & 1.292 & \\
$\left(\theta=90^{\circ}, \phi=0^{\circ}\right)$ & & 70 \\
$(22 \times \sqrt{3})$ reconstructed $\mathrm{Au}(111)$, hcp region & 1.362 & 90 \\
$(22 \times \sqrt{3})$ reconstructed $\mathrm{Au}(111)$, ridge region & 1.382 & 26 \\
$(22 \times \sqrt{3})$ reconstructed $\mathrm{Au}(111)$, fcc region & 1.318 & \\
\hline \hline
\end{tabular}

study. We have considered the three different regions of the reconstruction, which have been labeled "hcp-," "ridge-," and "fcc-" region in Ref. 79. Our focus is on the regions around the (threefold) sites that correspond to the extrema of the $\mathrm{H}$ atom adsorption energies (at $x=[25.4,38.3,58.4] \AA$ ) for the "hcp-," "ridge-," and "fcc-" region in the top panel of Figure 4 of Ref. 79, respectively. We transfer the minimum barrier geometry from the PBE-vdW-DF bridge-to-top configuration $\left(r_{b}=1.419 \AA, Z_{b}=1.479 \AA\right.$, see Table $\mathrm{S} 3$ of the supplementary material) to equivalent bridge-site configurations closest to these aforementioned three hollow sites by using the corresponding locally distorted surface lattice vectors. The results are given in Table V. With the reconstruction-induced distortion of the surface being the most (least) pronounced in the ridge (fcc) region (see bottom panel of Fig. 2 in Ref. 79), it is not surprising that we find the largest (smallest) differences $\Delta E_{b}$ to the barrier height (90 and $20 \mathrm{meV}$, respectively) on the un-reconstructed surface in these areas, with the barriers being higher on the reconstructed surface.

We note that these differences are of the same magnitude as those given by different density functionals on the unreconstructed $\mathrm{Au}(111)$ surface for this configuration and might deserve further attention in future work, when mapping of entire potential energy surfaces for dynamics calculations is computationally possible for the reconstructed surface. By neglecting the effect of the reconstruction, we might underestimate the dynamical barrier heights (see below) for dissociation of $\mathrm{H}_{2}$ on $\mathrm{Au}(111)$ by approximately $50 \mathrm{meV}$, i.e., by about $1 \mathrm{kcal} / \mathrm{mol}(\approx 43 \mathrm{meV})$ or more.

\section{B. Dynamics results}

Reaction probabilities are presented as a function of incidence energy $E_{i}$ in Figure 4(a) for all functionals used in this study and for $\mathrm{H}_{2}$ in its $(v=0, J=0)$ state, and for the $(v=0, J=3),(v=1, J=3)$, and $(v=2, J=3)$ states for the SRP48 and optPBE-vdW-DF functionals in Figures 4(b) and 4(c), respectively. The reaction probability curves computed with the PBE-vdW-DF, optPBE-vdW-DF, and SRP48 functionals for $(v=0, J=0)$ are rather similar, and are straddled by the reaction probability curves computed with the PBE and RPBE functionals. Similar findings apply to other rovibrational states. The reaction probabilities computed with the RPBE-vdW-DF functional are even smaller than the 


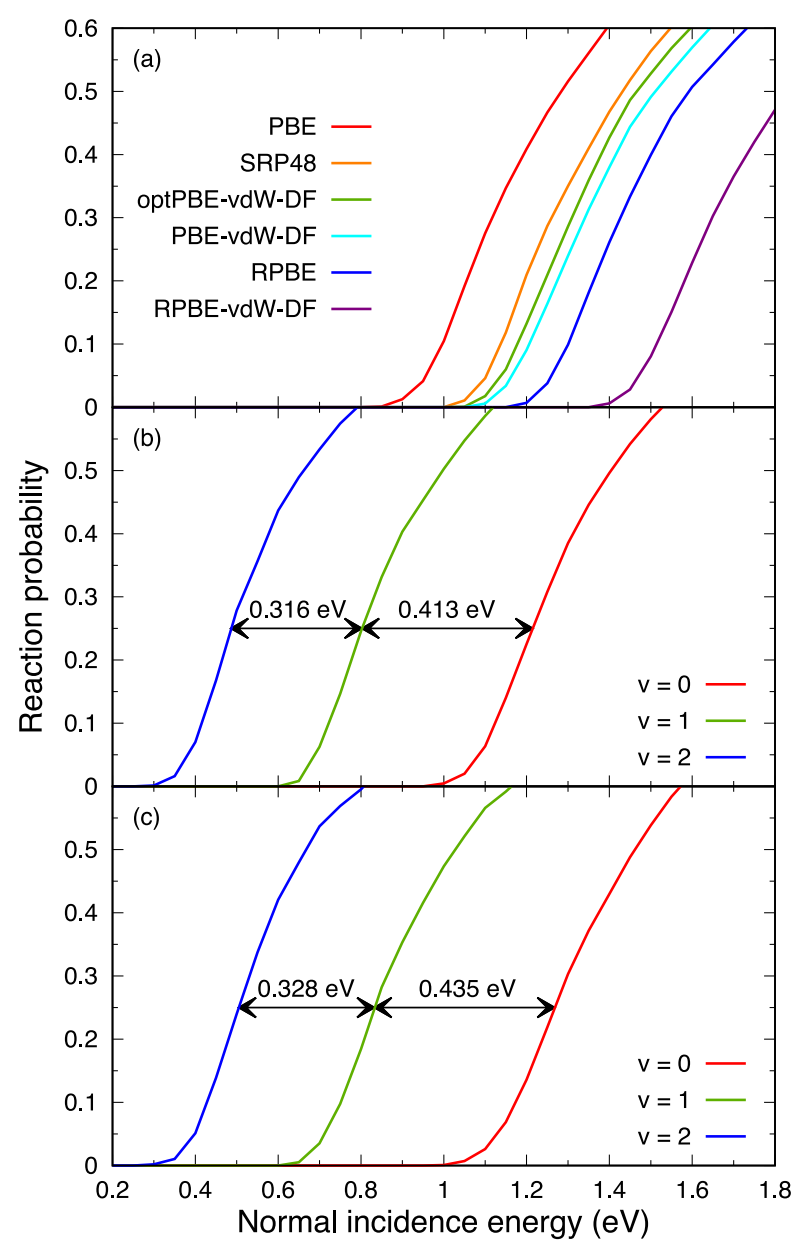

FIG. 4. Reaction probabilities as a function of incidence energy $E_{i}$ for all functionals used in this study and for $\mathrm{H}_{2}$ in its $(\mathrm{v}=0, \mathrm{j}=0)$ state (a), and for the $(v=0, J=3),(v=1, J=3)$, and $(v=2, J=3)$ states for the SRP48 (b) and optPBE-vdW-DF (c) functionals, respectively. Horizontal arrows and the numbers above these indicate the energy spacings between the reaction probability curves for the $(v, J=3)$ states, for a reaction probability equal to 0.25 .

RPBE reaction probabilities, reflecting the very high barriers obtained with RPBE-vdW-DF. Note: It might seem odd that the RPBE-vdW-DF functional yields higher barriers than the RPBE functional, and likewise that the PBE-vdW-DF functional yields higher barriers than the PBE functional. However, one should note that the Rutgers-Chalmers vdWDF functional ${ }^{80}$ is not just a functional that adds the attractive London dispersion van der Waals interaction to the potential. Rather, this functional is a general purpose correlation functional based on second order perturbation theory, ${ }^{107}$ which replaces the PBE correlation functional and leads to overall different results for the correlation energy. As a result, its use can lead to higher barriers, and this then simply reflects overall differences between the correlation energy obtained with vdW-DF and with PBE correlation. Here, one should keep in mind that at the short molecule-surface distance where the minimum barrier is located (about $1.2 \AA$ for the example of $\mathrm{H}_{2}+\mathrm{Cu}(111)^{19}$ ), the computed correlation energy corresponds to strongly overlapping charge clouds, whereas the van der Waals well minimum occurs at much larger distances (about $3.5 \AA$ for the $\mathrm{H}_{2}+\mathrm{Cu}(111)$ example

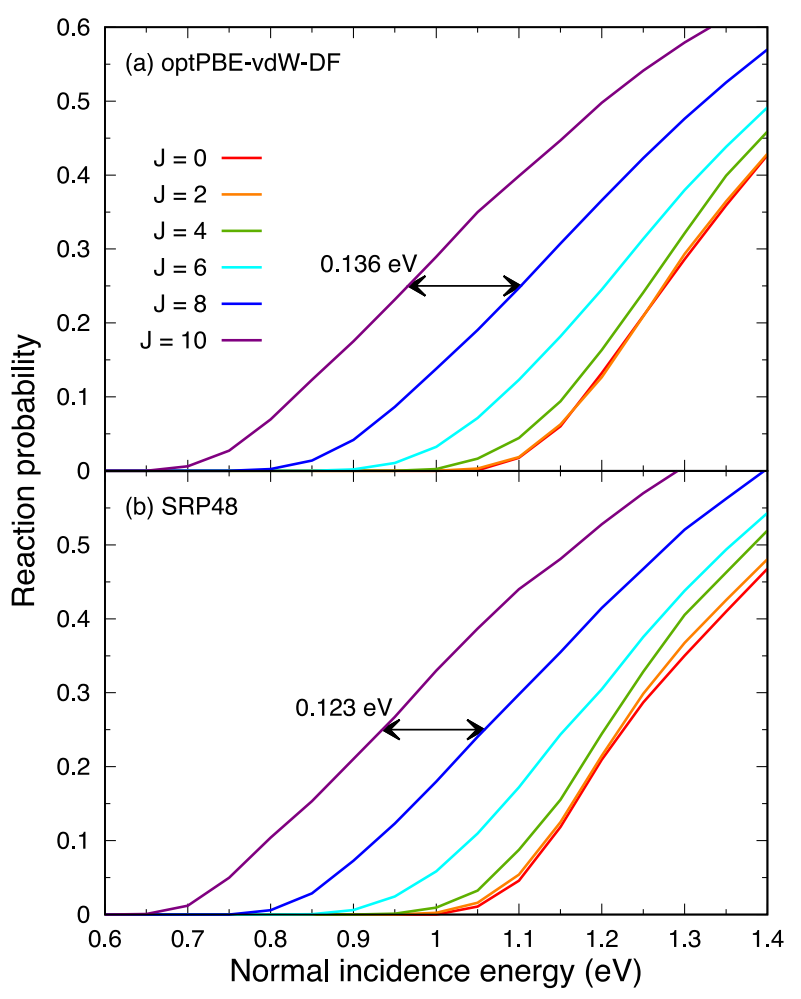

FIG. 5. Reaction probabilities as a function of incidence energy $E_{i}$ and for $\mathrm{H}_{2}$ in its $(v=0, J)$ state with $J$ even and $0 \leq J \leq 10$. Horizontal arrows and the numbers above it indicate energy spacings between the reaction probability curves for the $(v, J=8,10)$ states, for a reaction probability equal to 0.25 . Results obtained with the optPBE-vdW-DF (SRP48) functional are shown in panel a (b).

Ref. 108). For the latter case, where the charge clouds do not, or hardly, overlap, the interaction energy indeed shows the expected behavior, with functionals containing vdW-DF correlation showing a much more attractive interaction with the surface than functionals containing PBE correlation for the example given (see Figure 1 of Ref. 108).

Reaction probabilities computed with the SRP48 functional for the $(v=0, J)$ states with $J$ even and in the range 0-10 are shown in Fig. 5(b). The calculations with this functional predict that the reaction probability increases monotonically with $J$. This is at odds with experimental results for $\mathrm{H}_{2}+\mathrm{Cu}(111)^{52}$ and $\mathrm{D}_{2}+\mathrm{Cu}(111),{ }^{53}$ which show that, going from $J=0$ to higher $J$, the reactivity first decreases with $J$ up $J=4$ or 5 and then increases with $J$. However, calculations on $\mathrm{H}_{2}+\mathrm{Cu}(111)^{19}$ and $\mathrm{D}_{2}+\mathrm{Cu}(111)^{19,20}$ show the same monotonic trend as here found for $\mathrm{Au}(111)$. For $\mathrm{H}_{2}$ on $\mathrm{Cu}(111)$, the experimental trend in $J$ is thought to reflect the late barrier for reaction. At low $J$, increasing $J$ hinders the reaction because while traversing the narrow bottleneck to reaction, the molecule might rotate out of its most favorable orientation to react when rotating faster. ${ }^{52}$ At high $J$, increasing $J$ promotes the reaction because the rotational energy can be released to motion along the reaction coordinate, while the bond stretches towards the transition state for constant $J .^{52}$ A similar behavior might be expected for $\mathrm{H}_{2}+\mathrm{Au}(111)$, which also exhibits a late barrier. It is not yet clear why the delicate balance observed in experiments is not reproduced in calculations for $\mathrm{H}_{2}+\mathrm{Cu}(111)$, but it is 
perhaps not surprising that the calculations for the late barrier reaction $\mathrm{H}_{2}+\mathrm{Au}(111)$ show the same trend as calculations for the late barrier $\mathrm{H}_{2}+\mathrm{Cu}(111)$ reaction. The dependence of the reactivity on $J$ is not quite as monotonic for the optPBE-vdW-DF functional, for which $J=2$ and $J=0 \mathrm{H}_{2}$ exhibit more or less the same reactivity (Fig. 5(a)), albeit the difference observed with the behavior of the $J=2$ and $J=0$ curves obtained with the SRP48 functional is rather small. Note, however, that calculations on $\mathrm{H}_{2}+\mathrm{Cu}(111)$ also found a less monotonic dependence of the reaction probability on $J$ at low $J$ with optPBE-vdW-DF than with SRP48 (see Figure 6 of Ref. 82).

Reaction probabilities for $\mathrm{D}_{2}$ in its $(v=0, J=0)$ state and for $\mathrm{D}_{2}$ in its $(v, J=2)$ states with $v=0$-2 are presented in Figure 6. For one and the same functional, for $(v=0, J=0)$ $\mathrm{D}_{2}$, the reaction threshold energy is at a somewhat higher incidence energy than for $(v=0, J=0) \mathrm{H}_{2}$, which is a zero-point effect: ${ }^{109-111} \mathrm{H}_{2}$ has more energy in zero-point vibrational motion, so more of this energy can be converted to motion along the reaction coordinate, helping to traverse the barrier. This effect can only be recovered with quasi-classical

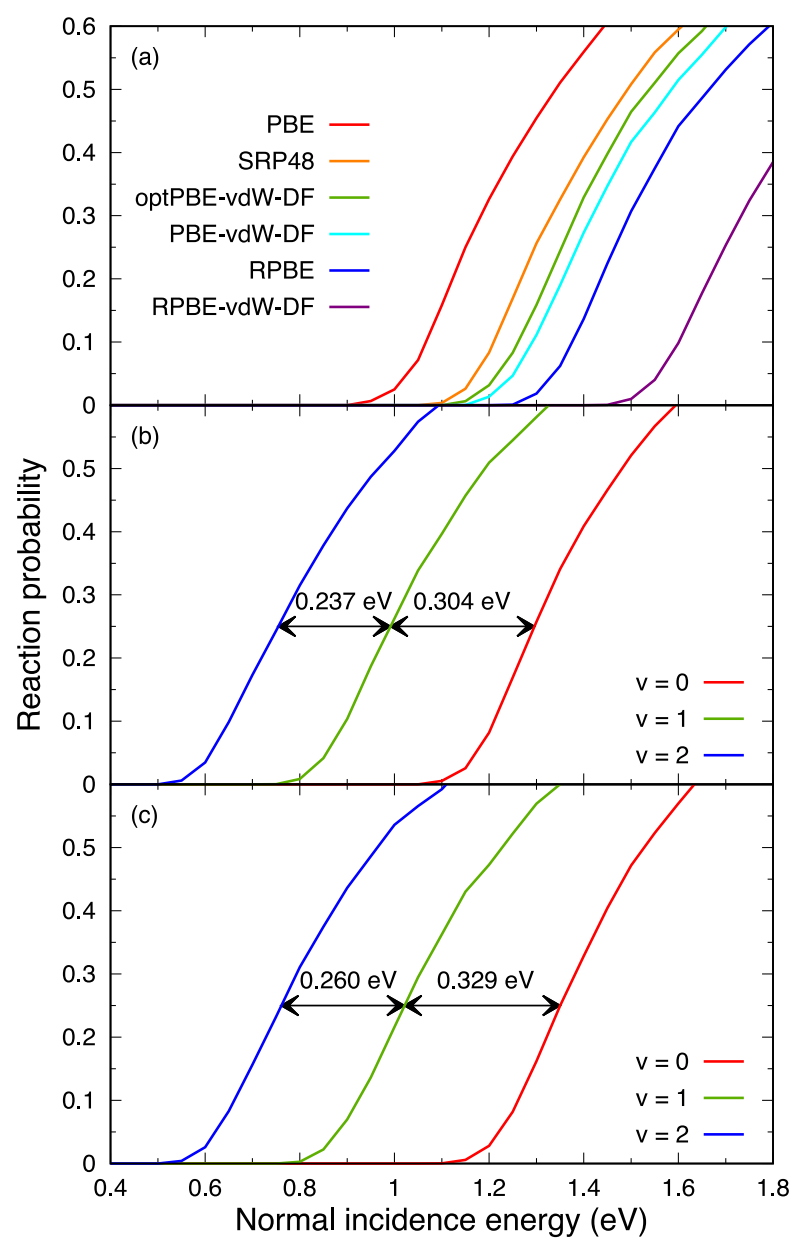

FIG. 6. Reaction probabilities as a function of incidence energy $E_{i}$ for all functionals used in this study and for $\mathrm{D}_{2}$ in its $(v=0, J=0)$ state (a), and for the $(v=0, J=2),(v=1, J=2)$, and $(v=2, J=2)$ states for the SRP48 (b) and optPBE-vdW-DF (c) functionals, respectively. Horizontal arrows and the numbers above these indicate the energy spacings between the reaction probability curves for the $(v, J=2)$ states, for a reaction probability equal to 0.25 .

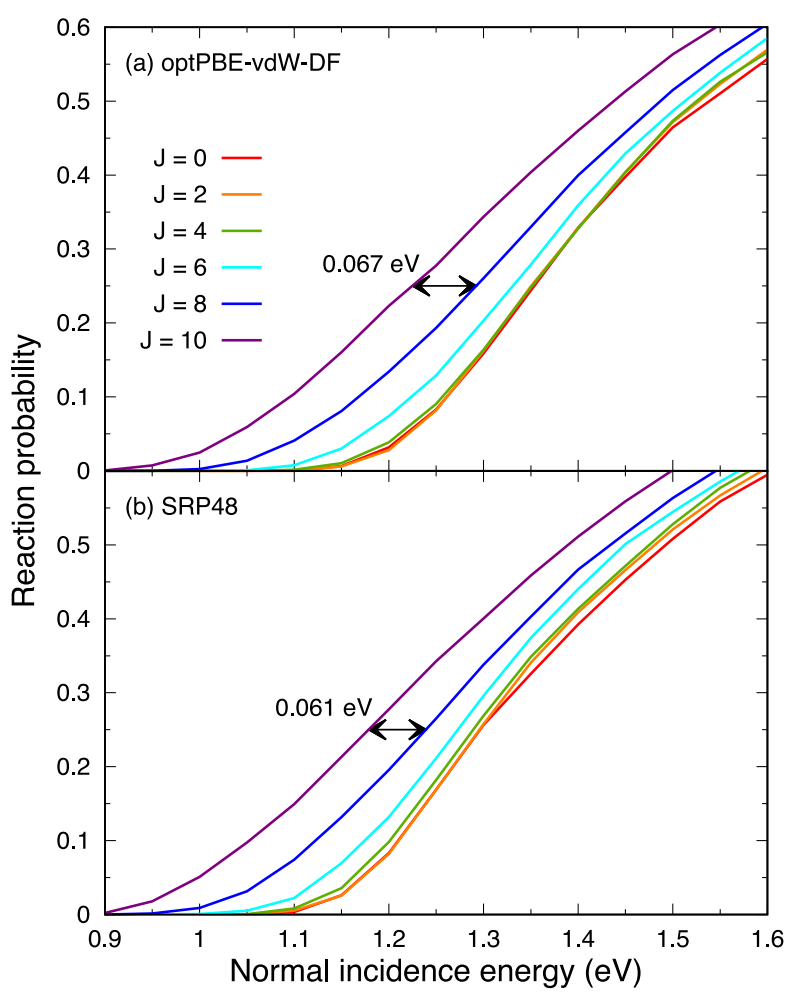

FIG. 7. Reaction probabilities as a function of incidence energy $E_{i}$ and for $\mathrm{D}_{2}$ in its $(v=0, J)$ state with $\mathrm{J}$ even and $0 \leq J \leq 10$. Horizontal arrows and the numbers above it indicate energy spacings between the reaction probability curves for the $(v, J=8,10)$ states, for a reaction probability equal to 0.25 . Results obtained with the optPBE-vdW-DF (SRP48) functional are shown in panel a (b).

dynamics: with the static surface approximation, results for $\mathrm{H}_{2}$ and $\mathrm{D}_{2}$ should be identical with a purely classical approach for the same incident energy and $(v=0, j=0)$, as discussed by Gross and Scheffler. ${ }^{112}$ Regarding the order of the reactivity, the trends obtained with the different functionals are the same as discussed earlier for $\mathrm{H}_{2}$ (Fig. 4). Reaction probabilities computed with the SRP48 functional for the $(v=0, J)$ states with $J$ even and in the range 0-10 are shown for $\mathrm{D}_{2}$ in Fig. 7(b). The same monotonic dependence of the reaction probability on $J$ is found as for $\mathrm{H}_{2}$ (Figure $5(\mathrm{~b})$ ). As for $\mathrm{H}_{2}$, for $\mathrm{D}_{2}$ the dependence of the reactivity on $J$ is not as monotonic for the optPBE-vdW-DF functional, for which $J=2$ and $J=0 \mathrm{H}_{2}$ exhibit more or less the same reactivity (Fig. 7(a)).

Vibrational and rotational efficacies are collected in Table VI for both $\mathrm{H}_{2}$ and $\mathrm{D}_{2}$. The optPBE-vdW-DF values for the vibrational efficacies come out somewhat larger than the SRP48 values, in agreement with earlier findings for $\mathrm{D}_{2}+\mathrm{Cu}(111) .{ }^{82}$ The vibrational efficacy $\eta_{v=0 \rightarrow 1}$ computed for $\mathrm{D}_{2}+\mathrm{Au}(111)$ (0.83 and 0.90 with SRP48 and optPBE-vdW$\mathrm{DF}$, respectively) is similar to that computed for $\mathrm{D}_{2}+\mathrm{Ag}(111)$ with the PBE functional (0.90). ${ }^{29}$ The values computed for $\eta_{v=0 \rightarrow 1}$ for $\mathrm{D}_{2}+\mathrm{Au}(111)$ are, however, much larger than those computed for $\mathrm{D}_{2}+\mathrm{Cu}(111)$ (0.65 for SRP48 and 0.71 for optPBE-vdW-DF, respectively). ${ }^{82}$ These trends reflect the difference in the lateness of the barrier between $\mathrm{H}_{2}+\mathrm{Cu}(111)$ and $\mathrm{H}_{2}+\mathrm{Au}(111)$ (barriers much later on $\mathrm{Au}$ ), and the similarity in the lateness of the barrier for $\mathrm{H}_{2}+\mathrm{Ag}(111)$ and $\mathrm{Au}(111)$, as discussed in Section III A. The decreased 
TABLE VI. Vibrational and rotational efficacies computed for $\mathrm{H}_{2}$ and $\mathrm{D}_{2}$ $+\mathrm{Au}(111)$ on the basis of the SRP48 PES and the optPBE-vdW-DF PES, where the latter are given in brackets.

\begin{tabular}{lcc}
\hline \hline Efficacy & $\mathrm{H}_{2}+\mathrm{Au}(111)$ & $\mathrm{D}_{2}+\mathrm{Au}(111)$ \\
\hline$\eta_{v=0 \rightarrow 1}$ & $0.81(0.86)$ & $0.83(0.90)$ \\
$\eta_{v=1 \rightarrow 2}$ & $0.65(0.68)$ & $0.67(0.74)$ \\
$\eta_{r o t}$ & $0.58(0.64)$ & $0.50(0.56)$ \\
\hline \hline
\end{tabular}

efficacy of vibration to promote reaction with increasing $v$ is a common observation in studies of activated dissociation of $\mathrm{D}_{2}{ }^{20}$ and $\mathrm{CH}_{4}{ }^{113}$ alike. The rotational efficacies are mainly presented as predictions for experiment.

We have also analysed the dynamics to see whether the reaction occurs in a direct or an indirect fashion, and whether the reaction occurs predominantly at specific surface sites. For this, the trajectories calculated for $(v=0, J=0)$ $\mathrm{H}_{2}$ were considered, as computed with the optPBE-vdW-DF functional. We first looked at the probability for scattering back to the gas phase. The total value changes from 1 at the lowest incidence energy $(0.05 \mathrm{eV})$ to 0.18 at the highest incidence energy studied $(2.1 \mathrm{eV})$. Over this entire energy range, the probability for indirect scattering (scattering trajectory exhibiting more than one turning point in Z) did not exceed 0.04. This already strongly suggests that also reactive scattering is primarily direct, i.e., occurs without the molecule performing bounces on the surface prior to reaction. This is corroborated by evaluating the probability that the molecule reacts while exhibiting one or more bounces (two or more inner turning points in $\mathrm{Z}$ ) before the trajectory is ended because the $\mathrm{H}-\mathrm{H}$ distance reaches the critical value at which the trajectory is counted as reaction. Over the entire energy range considered, this probability did not exceed 0.06 , while the reaction probability rises to about 0.82 . The reaction therefore occurs on a fast time scale, and non-adiabatic effects have to act efficiently on this short time scale in order to strongly affect the probability of the molecule to react. Similar results were obtained with the SRP48 functional.

To analyse whether the reaction is site-specific, the area of the surface unit cell was assigned to top, bridge, and hollow sites (fcc and hcp, see Fig. 1) in a reasonable way (as done in Figure 2 in recent work on $\mathrm{H}_{2}+\mathrm{CO}$ precovered $\mathrm{Ru}(0001)$, not making any distinction between sites closest and farthest away from pre-adsorbed $\mathrm{CO}$ in that work). Only at the very lowest energy was a clear preference for reaction site found. For instance, at $1.1 \mathrm{eV}$ the probability of reaction at the bridge site was more than 4 (6) times larger than at the hollow sites (top site). Already at $1.15 \mathrm{eV}$ the hollow sites were slightly more reactive than the bridge site (by only a small margin, and the bridge site remains the most reactive one if its greater associated surface area is taken into account) and the top site (by a factor 1.5). This difference in reactivity between the sites holds up to about $1.5 \mathrm{eV}$, and at higher incidence energies this difference almost disappears, the sites becoming almost equally reactive. It follows that, if one is interested in non-adiabatic effects, one should in principle consider non-adiabatic couplings at all sites, because all sites are, to within a good approximation, equally reactive in the adiabatic dynamics. Similar results were obtained with the SRP48 functional.

\section{Fits to and features of reaction probability curves}

For ease of use in applications where time-of-flight spectra for associative desorption are computed from dissociation probability curves by invoking detailed balance, ${ }^{20}$ we have attempted to fit the $\mathrm{H}_{2}+\mathrm{Au}(111)$ reaction probabilities computed with the SRP48 and optPBE-vdW-DF functionals to a suitable form. The four-parameter generalized logistics functional used successfully in applications on $\mathrm{D}_{2}+\mathrm{Cu}(111)^{20}$ proved less useful for the present $\mathrm{H}_{2}+\mathrm{Au}(111)$ results. For the latter system, better results were obtained by fitting the reaction probability curves to the five-parameter curve (FPC), ${ }^{20}$

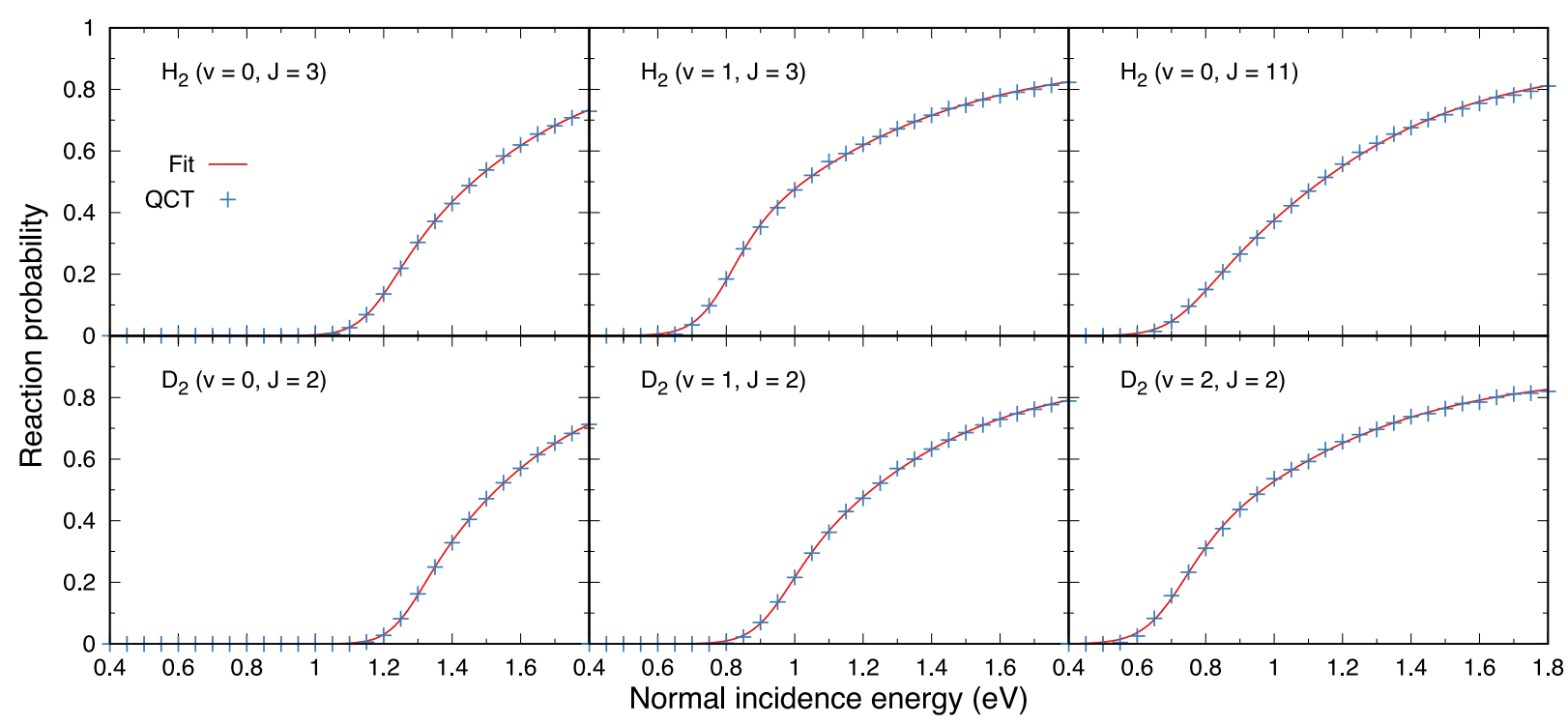

FIG. 8. Reaction probabilities computed with the SRP48 functional, and the fits of the reaction probability curves through these data on the basis of the FPC expression, (Eq. (4)), are shown as a function of $E_{i}$ for the three $\mathrm{H}_{2}$ and the three $\mathrm{D}_{2}$ rovibrational states indicated. 


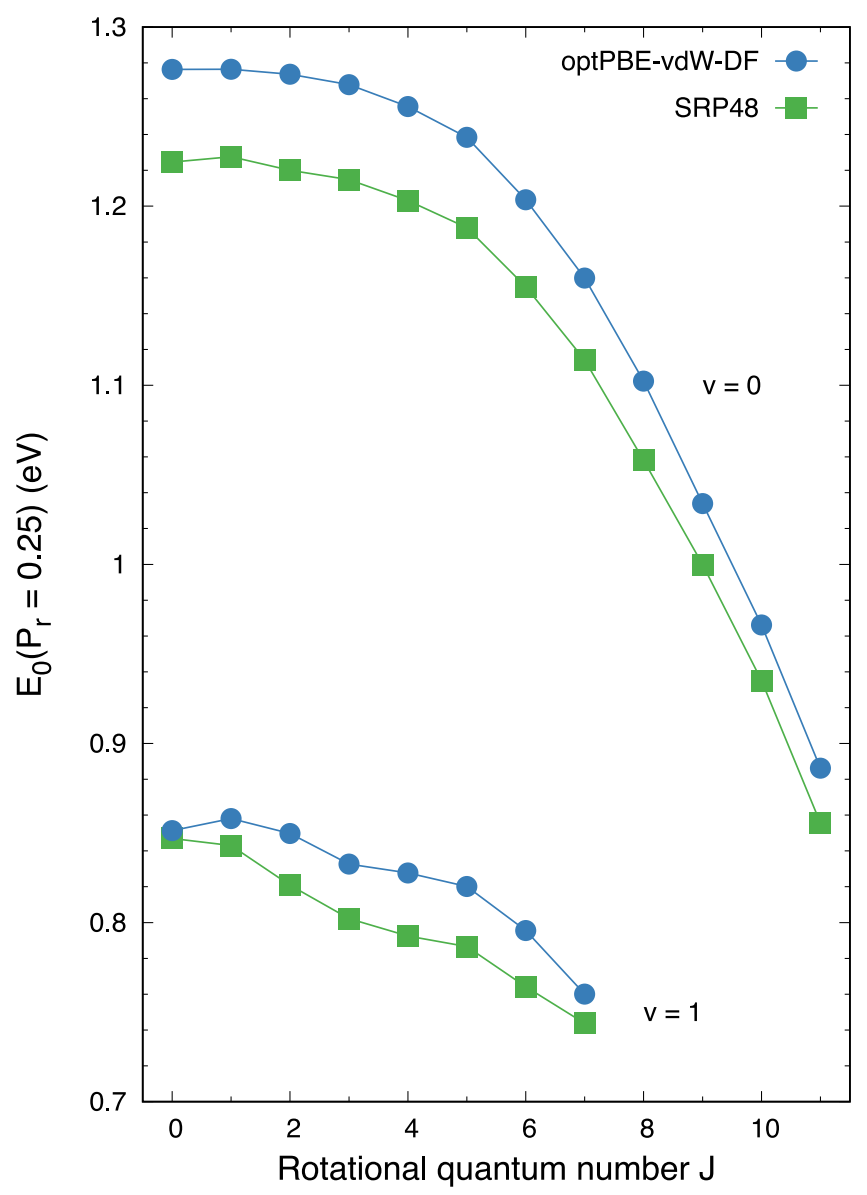

FIG. 9. The dynamical barrier height $E_{0}$ computed with the optPBE-vdWDF (blue circles) and SRP48 (green squares) functionals is shown as a function of $J$, for $\mathrm{H}_{2}+\mathrm{Au}(111)$, for $v=0$ and 1 .

$P_{r}\left(E_{i}\right)=A \exp \left[-\exp \left(-\frac{E_{i}-E_{0}^{\prime}}{W^{\prime}}\right)\right] /\left[1+\exp \left(-\frac{E_{i}-E_{0}^{\prime \prime}}{W^{\prime \prime}}\right)\right]$

As shown in Fig. 8, this expression allows excellent fits of the reaction probabilities computed with the QCT method for $(v, J) \mathrm{H}_{2}+\mathrm{Au}(111)$ for $(v=0-1, J=3)$ and for $(v=0, J$ $=11$ ) using a SRP48 PES, and similar results were obtained for other rovibrational states and for the optPBE-vdW-DF functional. Similar results were also obtained for $(v=0-2, J$ = 2) $\mathrm{D}_{2}+\mathrm{Au}(111)$ (see also Fig. 8). The parameters obtained for the $(v, J) \mathrm{H}_{2}$ and $\mathrm{D}_{2}$ states, studied on the basis of the SRP48, optPBE-vdW-DF, and PBE functionals, are tabulated in Tables S4-S9 of the supplementary material. There, we also provide the reaction probabilities computed for $\mathrm{D}_{2}+\mathrm{Au}(111)$ with the SRP48 and optPBE-vdW-DF functionals. In comparisons of our calculated reaction probabilities with experiments performed for associative desorption from a hot $\mathrm{Au}(111)$ surface, one should keep in mind that the static surface approximation used here will most likely underestimate the widths of the computed reaction probability curves for a hot surface (i.e., $T_{s} \geq 900 \mathrm{~K}$ ). ${ }^{32,114}$

The energy constants in the FPC curve do not provide much physical insight. A more useful measure of the reactivity is the value of the incidence energy for which the reaction probability first attains a specific value, chosen to be 0.25 in this work. Such a value of the incidence energy can be

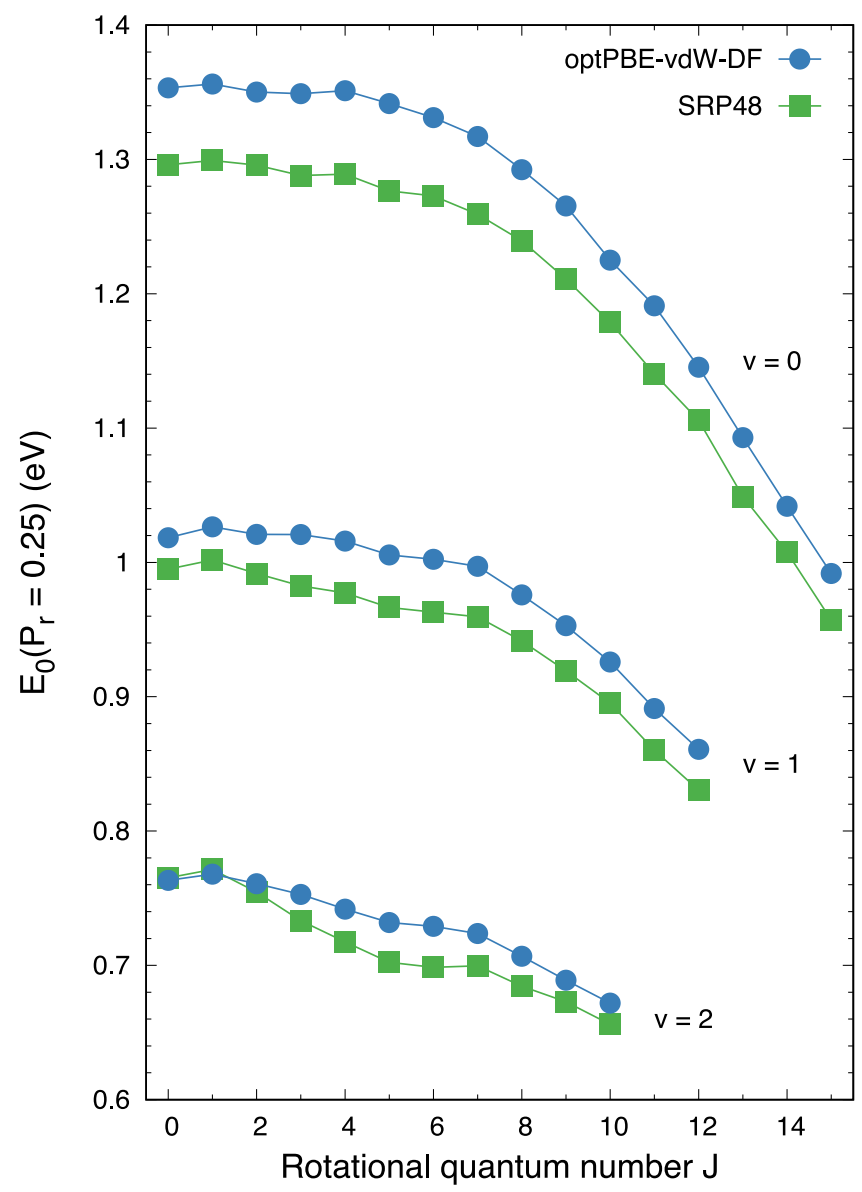

FIG. 10. The dynamical barrier height $E_{0}$ computed with the optPBE-vdWDF (blue circles) and SRP48 (green squares) functionals is shown as a function of $J$, for $\mathrm{D}_{2}+\mathrm{Au}(111)$, for $v=0,1$, and 2 .

denoted as $E_{0}$, and called the dynamical barrier height. It is plotted as a function of $J$ for $v=0$ and $1 \mathrm{H}_{2}$ in Figure 9, and for $v=0,1$, and $2 \mathrm{D}_{2}$ in Figure 10. As a function of $J$, the computed $E_{0}$ values display the trend of a monotonic decrease with $J$ as usually found in dynamics calculations based on DFT PESs, ${ }^{19,20,64}$ although in some cases observed here $E_{0}$ first goes up with $J$ going from $J=0$ to 1 , after which it then decreases with increasing $J$. In contrast, experiments on $\mathrm{H}_{2}$ and $\mathrm{D}_{2}+\mathrm{Cu}(111)$ have usually shown $E_{0}$ to increase with $J$ for $J$ up to 4 or 5 , and then to decrease with increasing $J$. The experimental dependence of $E_{0}$ on $J$ for $\mathrm{H}_{2}+\mathrm{Au}(111)$ remains to be established.

\section{CONCLUSIONS}

We have performed calculations on the dissociative chemisorption of $\mathrm{H}_{2}$ on un-reconstructed $\mathrm{Au}(111)$. Together with the $\mathrm{H}_{2}+\mathrm{Ag}(111)$ system, due to its late barrier for dissociation, $\mathrm{H}_{2}+\mathrm{Au}(111)$ is among the best examples of $\mathrm{H}_{2}+$ metal surface systems for which the dissociative chemisorption could be considerably affected by dissipation of energy to the metal electrons while $\mathrm{H}_{2}$ travels to the late reaction barrier.

Minimum barrier geometries and potential energy surfaces (PESs) were computed for six density functionals, 
i.e., three GGA functionals using PBE correlation, and three functionals exhibiting GGA exchange and non-local correlation as used in the vdW-DF1 functional of Dion et al. ${ }^{80}$ Two of the functionals tested (SRP48 and optPBE-vdW-DF) have previously shown excellent performance on $\mathrm{H}_{2}$-metal surface systems. The six functionals tested yield minimum barrier heights in the range $1.15-1.6 \mathrm{eV}$, and very late barriers in the sense that the $\mathrm{H}-\mathrm{H}$ distances at the barrier geometries are not only larger than the equilibrium gas phase $\mathrm{H}_{2}$ bond distance but also larger than found for the barriers in the late barrier $\mathrm{H}_{2}+\mathrm{Cu}(111)$ system.

The PESs have been used in quasi-classical trajectory $(\mathrm{QCT})$ calculations of the initial $(\mathrm{v}, J)$ state-resolved reaction probability for several rovibrational states of $\mathrm{H}_{2}$ and $\mathrm{D}_{2}$. Our calculations may serve as predictions for state-resolved associative desorption experiments, from which initial stateresolved dissociative chemisorption probabilities can be extracted by invoking detailed balance. For this purpose, the reaction probabilities computed for several $\mathrm{H}_{2}$ and $\mathrm{D}_{2}$ rovibrational states with $v=0-2$ have been fitted to an analytical form for the SRP48, optPBE-vdW-DF, and PBE functionals, and the fits have been reported. The vibrational efficacy $\eta_{v=0 \rightarrow v=1}$ reported for $\mathrm{D}_{2}$ dissociating on un-reconstructed $\mathrm{Au}(111)$ (about 0.9) is similar to that found in earlier quantum dynamics calculations on $\mathrm{H}_{2}+\mathrm{Ag}(111),{ }^{29}$ but larger than found computationally and experimentally for $\mathrm{D}_{2}+\mathrm{Cu}(111)$. With both functionals tested, the reactivity of $\mathrm{H}_{2}$ and $\mathrm{D}_{2}$ exhibits an almost monotonic dependence on the rotational quantum number $J$. This is at odds with experiments on $\mathrm{H}_{2}$ and $\mathrm{D}_{2}+\mathrm{Cu}(111)$, which predict that the reactivity should first decrease with increasing $J$ up to $J=4$ or 5, and then decrease with increasing $J$.

A limited set of test calculations employing the PBEvdW-DF functional have been performed on the dissociation barrier of $\mathrm{H}_{2}$ on herringbone-reconstructed $\mathrm{Au}(111)$. These tests predict that the dissociation barrier on the reconstructed surface is higher by values in the range $20-90 \mathrm{meV}$ when compared to the un-reconstructed surface. These results suggest that, for chemical accuracy (i.e., reaction probability curves accurate to within energy shifts of $1 \mathrm{kcal} / \mathrm{mol}$ ), the herringbone reconstruction of $\mathrm{Au}(111)$ should be modeled when performing calculations with the aim of reproducing experiments.

\section{SUPPLEMENTARY MATERIAL}

See the supplementary material for Tables S1-S9, tabulated computed reaction probabilities for $\mathrm{H}_{2}, \mathrm{D}_{2}+\mathrm{Au}(111)$ based on the SRP48 and optPBE-vdW-DF functionals, and the potential data needed to construct the six potential energy surfaces used in this work.

\section{ACKNOWLEDGMENTS}

This work was supported financially by the Nederlandse organisatie voor Wetenschappelijk onderzoek (NWO-CW), by the European Research Council through an ERC-2013 advanced grant (No. 338580), and with computer time granted by NWO-EW. We are grateful for useful discussions with
Professor Daniel Auerbach and Professor Alec Wodtke, with Dr. Dirk Schwarzer and Dr. Quan Shuai, and with Mr. Sven Kaufmann. We thank Dr. Francesco Nattino for providing us with the results of a Bader charge analysis for $\mathrm{H}_{2}+\mathrm{Cu}(111)$.

${ }^{1}$ J. D. White, J. Chen, D. Matsiev, D. J. Auerbach, and A. M. Wodtke, Nature 433, 503 (2005).

${ }^{2}$ P. Nieto, E. Pijper, D. Barredo, G. Laurent, R. A. Olsen, E. J. Baerends, G. J. Kroes, and D. Farías, Science 312, 86 (2006).

${ }^{3}$ M. Lindenblatt and E. Pehlke, Phys. Rev. Lett. 97, 216101 (2006).

${ }^{4}$ G. J. Kroes, Science 321, 794 (2008).

${ }^{5}$ J. I. Juaristi, M. Alducin, R. Díez Muiño, H. F. Busnengo, and A. Salin, Phys. Rev. Lett. 100, 116102 (2008)

${ }^{6}$ N. H. Nahler, J. D. White, J. Larue, D. J. Auerbach, and A. M. Wodtke, Science 321, 1191 (2008).

${ }^{7}$ A. C. Luntz, I. Makkonen, M. Persson, S. Holloway, D. M. Bird, and M. S. Mizielinski, Phys. Rev. Lett. 102, 109601 (2009).

${ }^{8}$ N. Shenvi, S. Roy, and J. C. Tully, Science 326, 829 (2009).

${ }^{9}$ E. Hasselbrink, Science 326, 809 (2009).

${ }^{10}$ O. Bünermann, H. Y. Jiang, Y. Dorenkamp, A. Kandratsenka, S. M. Janke, D. J. Auerbach, and A. M. Wodtke, Science 350, 1346 (2015).

${ }^{11}$ S. N. Maximoff and M. P. Head-Gordon, Proc. Natl. Acad. Sci. U. S. A. 106, 11460 (2009).

${ }^{12}$ X. Z. Ji, A. Zuppero, J. M. Gidwani, and G. A. Somorjai, J. Am. Chem. Soc. 127, 5792 (2005).

${ }^{13}$ J. Y. Park, J. R. Renzas, A. M. Contreras, and G. A. Somorjai, Top. Catal. 46, 217 (2007)

${ }^{14}$ M. Pavanello, D. J. Auerbach, A. M. Wodtke, M. Blanco-Rey, M. Alducin, and G. J. Kroes, J. Phys. Chem. Lett. 4, 3735 (2013).

${ }^{15}$ A. J. Komrowski, J. Z. Sexton, A. C. Kummel, M. Binetti, O. Weisse, and E. Hasselbrink, Phys. Rev. Lett. 87, 246103 (2001).

${ }^{16}$ B. Gergen, H. Nienhaus, W. H. Weinberg, and E. W. McFarland, Science 294, 2521 (2001)

${ }^{17}$ H. Nienhaus, Surf. Sci. Rep. 45, 1 (2002).

${ }^{18}$ G. J. Kroes, Phys. Chem. Chem. Phys. 14, 14966 (2012).

${ }^{19}$ C. Díaz, E. Pijper, R. A. Olsen, H. F. Busnengo, D. J. Auerbach, and G. J. Kroes, Science 326, 832 (2009).

${ }^{20}$ F. Nattino, A. Genova, M. Guijt, A. S. Muzas, C. Díaz, D. J. Auerbach, and G. J. Kroes, J. Chem. Phys. 141, 124705 (2014).

${ }^{21}$ A. C. Luntz and M. Persson, J. Chem. Phys. 123, 074704 (2005).

${ }^{22}$ G. Füchsel, S. Schimka, and P. Saalfrank, J. Phys. Chem. A 117, 8761 (2013).

${ }^{23}$ A. S. Muzas, J. I. Juaristi, M. Alducin, R. Diéz Muiño, G. J. Kroes, and C. Díaz, J. Chem. Phys. 137, 064707 (2012).

${ }^{24}$ Y. H. Huang, C. T. Rettner, D. J. Auerbach, and A. M. Wodtke, Science 290, 111 (2000).

${ }^{25}$ M. Cízek, J. Horácek, and W. Domcke, J. Phys. B 31, 2571 (1998).

${ }^{26}$ H. B. Michaelson, J. Appl. Phys. 48, 4729 (1977).

${ }^{27}$ B. Hammer and J. K. Nørskov, Nature 376, 238 (1995).

${ }^{28}$ F. Libisch, J. Cheng, and E. A. Carter, Z. Phys. Chem. 227, 1455 (2013).

${ }^{29}$ B. Jiang and H. Guo, Phys. Chem. Chem. Phys. 16, 24704 (2014).

${ }^{30}$ B. Schindler, D. Diesing, and E. Hasselbrink, J. Chem. Phys. 134, 034705 (2011).

${ }^{31}$ B. Schindler, D. Diesing, and E. Hasselbrink, Z. Phys. Chem. 227, 1381 (2013).

${ }^{32}$ F. Nattino, C. Díaz, B. Jackson, and G. J. Kroes, Phys. Rev. Lett. 108, 236104 (2012).

${ }^{33}$ M. Blanco-Rey, J. I. Juaristi, R. Díez Muiño, H. F. Busnengo, G. J. Kroes, and M. Alducin, Phys. Rev. Lett. 112, 103203 (2014).

${ }^{34}$ S. Mukherjee, F. Libisch, N. Large, O. Neumann, L. V. Brown, J. Cheng, J. B. Lassiter, E. A. Carter, P. Nordlander, and N. J. Halas, Nano Lett. 13, 240 (2012).

${ }^{35}$ T. V. Choudhary, C. Sivadinarayana, A. K. Datye, D. Kumar, and D. W. Goodman, Catal. Lett. 86, 1 (2003).

${ }^{36}$ C. Mohr, H. Hofmeister, J. Radnik, and P. Claus, J. Am. Chem. Soc. 125, 1905 (2003).

${ }^{37}$ A. Corma and P. Serna, Science 313, 332 (2006).

${ }^{38}$ T. Fujitani, I. Nakamura, T. Akita, M. Okumura, and M. Haruta, Angew. Chem. 121, 9679 (2009).

${ }^{39}$ S. A. Varganov, R. M. Olson, M. S. Gordon, G. Mills, and H. Metiu, J. Chem. Phys. 120, 5169 (2004).

${ }^{40}$ M. Okumura, Y. Kitagawa, M. Haruta, and K. Yamaguchi, Appl. Catal., A 291, 37 (2005). 
${ }^{41}$ A. Corma, M. Boronat, S. González, and F. Illas, Chem. Commun. 2007, 3371.

${ }^{42}$ S. Csonka, A. Halbritter, G. Mihály, E. Jurdik, O. I. Shklyarevskii, S. Speller, and H. van Kempen, Phys. Rev. Lett. 90, 116803 (2003).

${ }^{43}$ P. Jelínek, R. Pérez, J. Ortega, and F. Flores, Phys. Rev. Lett. 96, 046803 (2006).

${ }^{44}$ W.-Y. Yu, G. M. Mullen, and C. B. Mullins, J. Phys. Chem. C 117, 19535 (2013).

${ }^{45}$ N. Takehiro, P. Liu, A. Bergbreiter, J. K. Nørskov, and R. J. Behm, Phys. Chem. Chem. Phys. 16, 23930 (2014).

${ }^{46}$ M. Pan, Z. D. Pozun, W.-Y. Yu, G. Henkelman, and C. B. Mullins, J. Phys. Chem. Lett. 3, 1894 (2012).

${ }^{47}$ M. Pan, D. W. Flaherty, and C. B. Mullins, J. Phys. Chem. Lett. 2, 1363 (2011).

${ }^{48}$ A. Sault, R. J. Madix, and C. T. Campbell, Surf. Sci. 169, 347 (1986).

${ }^{49}$ G. J. Kroes, M. Pavanello, M. Blanco-Rey, M. Alducin, and D. J. Auerbach, J. Chem. Phys. 141, 054705 (2014).

${ }^{50}$ S. M. Janke, M. Pavanello, G. J. Kroes, D. Auerbach, A. M. Wodtke, and A. Kandratsenka, Z. Phys. Chem. 227, 1467 (2013).

${ }^{51}$ H. F. Berger, M. Leisch, A. Winkler, and K. D. Rendulic, Chem. Phys. Lett. 175, 425 (1990).

${ }^{52}$ C. T. Rettner, H. A. Michelsen, and D. J. Auerbach, J. Chem. Phys. 102, 4625 (1995).

${ }^{53}$ H. A. Michelsen, C. T. Rettner, D. J. Auerbach, and R. N. Zare, J. Chem. Phys. 98, 8294 (1993).

${ }^{54}$ C. T. Rettner, D. J. Auerbach, and H. A. Michelsen, Phys. Rev. Lett. 68, 1164 (1992).

${ }^{55}$ A. Hodgson, P. Samson, A. Wight, and C. Cottrell, Phys. Rev. Lett. 78, 963 (1997).

${ }^{56}$ A. Hodgson, J. Moryl, P. Traversaro, and H. Zhao, Nature 356, 501 (1992).

${ }^{57}$ C. T. Rettner, D. J. Auerbach, and H. A. Michelsen, Phys. Rev. Lett. 68, 2547 (1992).

${ }^{58}$ C. T. Rettner, H. A. Michelsen, and D. J. Auerbach, Chem. Phys. 175, 157 (1993).

${ }^{59}$ A. Gross, B. Hammer, M. Scheffler, and W. Brenig, Phys. Rev. Lett. 73, 3121 (1994).

${ }^{60}$ J. Q. Dai and J. C. Light, J. Chem. Phys. 107, 1676 (1997).

${ }^{61}$ J. Q. Dai and J. C. Light, J. Chem. Phys. 108, 7816 (1998).

${ }^{62}$ M. F. Somers, S. M. Kingma, E. Pijper, G. J. Kroes, and D. Lemoine, Chem. Phys. Lett. 360, 390 (2002).

${ }^{63}$ S. Nave, D. Lemoine, M. F. Somers, S. M. Kingma, and G. J. Kroes, J. Chem. Phys. 122, 214709 (2005).

${ }^{64}$ C. Díaz, R. A. Olsen, D. J. Auerbach, and G. J. Kroes, Phys. Chem. Chem. Phys. 12, 6499 (2010).

${ }^{65}$ C. Díaz, R. A. Olsen, H. F. Busnengo, and G. J. Kroes, J. Phys. Chem. C 114, 11192 (2010)

${ }^{66}$ G. J. Kroes, C. Díaz, E. Pijper, R. A. Olsen, and D. J. Auerbach, Proc. Natl. Acad. Sci. U. S. A. 107, 20881 (2010).

${ }^{67}$ A. Mondal, M. Wijzenbroek, M. Bonfanti, C. Díaz, and G. J. Kroes, J. Phys. Chem. A 117, 8770 (2013).

${ }^{68}$ F. Healey, R. N. Carter, and A. Hodgson, Surf. Sci. 328, 67 (1995).

${ }^{69}$ M. J. Murphy and A. Hodgson, Surf. Sci. 368, 55 (1996).

${ }^{70}$ M. J. Murphy and A. Hodgson, Surf. Sci. 390, 29 (1997).

${ }^{71}$ M. J. Murphy and A. Hodgson, Phys. Rev. Lett. 78, 4458 (1997).

${ }^{72}$ F. Healey, R. N. Carter, G. Worthy, and A. Hodgson, Chem. Phys. Lett. 243, 133 (1995).

${ }^{73}$ C. Cottrell, R. N. Carter, A. Nesbitt, P. Samson, and A. Hodgson, J. Chem. Phys. 106, 4714 (1997).

${ }^{74}$ D. A. King and M. G. Wells, Proc. R. Soc. London, Ser. A 339, 245 (1974).
${ }^{75}$ J. P. Perdew, K. Burke, and M. Ernzerhof, Phys. Rev. Lett. 77, 3865 (1996).

${ }^{76}$ B. Hammer, L. B. Hansen, and J. K. Nørskov, Phys. Rev. B 59, 7413 (1999).

${ }^{77}$ A. R. Sandy, S. G. J. Mochrie, D. M. Zehner, K. G. Huang, and D. Gibbs, Phys. Rev. B 43, 4667 (1991).

${ }^{78}$ U. Harten, A. M. Lahee, J. P. Toennies, and C. Wöll, Phys. Rev. Lett. 54, 2619 (1985).

${ }^{79}$ F. Hanke and J. Björk, Phys. Rev. B 87, 235422 (2013).

${ }^{80}$ M. Dion, H. Rydberg, E. Schröder, D. C. Langreth, and B. I. Lundqvist, Phys. Rev. Lett. 92, 246401 (2004).

${ }^{81}$ J. Klimes, D. R. Bowler, and A. Michaelides, J. Phys.: Condens. Matter 22, 022201 (2010).

${ }^{82}$ M. Wijzenbroek, D. M. Klein, B. Smits, M. F. Somers, and G. J. Kroes, J. Phys. Chem. A 119, 12146 (2015).

${ }^{83}$ H. F. Busnengo, A. Salin, and W. Dong, J. Chem. Phys. 112, 7641 (2000).

${ }^{84}$ R. A. Olsen, H. F. Busnengo, A. Salin, M. F. Somers, G. J. Kroes, and E. J. Baerends, J. Chem. Phys. 116, 3841 (2002).

${ }^{85}$ M. Wijzenbroek and G. J. Kroes, J. Chem. Phys. 140, 084702 (2014).

${ }^{86}$ T. J. Frankcombe, M. A. Collins, and D. H. Zhang, J. Chem. Phys. 137, 144701 (2012).

${ }^{87}$ M. Karplus, R. N. Porter, and R. D. Sharma, J. Chem. Phys. 43, 3259 (1965).

${ }^{88}$ D. A. McCormack and G. J. Kroes, Chem. Phys. Lett. 296, 515 (1998).

${ }^{89}$ E. Pijper, M. F. Somers, G. J. Kroes, R. A. Olsen, E. J. Baerends, H. F. Busnengo, A. Salin, and D. Lemoine, Chem. Phys. Lett. 347, 277 (2001).

${ }^{90}$ C. C. Marston and G. C. Balint-Kurti, J. Chem. Phys. 91, 3571 (1989).

${ }^{91}$ J. Stoer and R. Burlisch, Introduction to Numerical Analysis (Springer, New York, 1980).

${ }^{92}$ G. Kresse and J. Furthmüller, Phys. Rev. B 54, 11169 (1996).

${ }^{93}$ G. Kresse and D. Joubert, Phys. Rev. B 59, 1758 (1999).

${ }^{94} \mathrm{G}$. Kresse and J. Hafner, J. Phys.: Condens. Matter 6, 8245 (1994).

${ }^{95}$ D. Vanderbilt, Phys. Rev. B 41, 7892 (1990).

${ }^{96}$ P. E. Blöchl, Phys. Rev. B 50, 17953 (1994).

${ }^{97}$ G. Román-Pérez and J. M. Soler, Phys. Rev. Lett. 103, 096102 (2009).

${ }^{98}$ B. N. Dutta and B. Dayal, Phys. Status Solidi 3, 473 (1963).

${ }^{99} \mathrm{R}$. Bader, Atoms in Molecules: A quantum Theory (Oxford University Press, New York, 1990).

${ }^{100}$ W. Tang, E. Sanville, and G. Henkelman, J. Phys.: Condens. Matter 21, 084204 (2009).

${ }^{101}$ E. Sanville, S. D. Kenny, R. Smith, and G. Henkelman, J. Comput. Chem. 28, 899 (2007).

${ }^{102}$ G. Henkelman, A. Arnaldsson, and H. Jónsson, Comput. Mater. Sci. 36, 254 (2006).

${ }^{103}$ M. Yu and D. R. Trinkle, J. Chem. Phys. 134, 064111 (2011).

${ }^{104}$ F. Libisch, C. Huang, P. L. Liao, M. Pavone, and E. A. Carter, Phys. Rev. Lett. 109, 198303 (2012).

${ }^{105}$ J. C. Polanyi, Science 236, 680 (1987).

${ }^{106}$ G. J. Kroes and C. Díaz, Chem. Soc. Rev. 45, 3658 (2016)

${ }^{107}$ K. Berland, V. R. Cooper, K. Lee, E. Schröder, T. Thonhauser, P. Hyldgaard, and B. I. Lundqvist, Rep. Prog. Phys. 78, 066501 (2015).

${ }^{108}$ K. Lee, A. K. Kelkkanen, K. Berland, S. Andersson, D. C. Langreth, E. Schröder, B. I. Lundqvist, and P. Hyldgaard, Phys. Rev. B 84, 193408 (2011).

${ }^{109}$ C. M. Chiang and B. Jackson, J. Chem. Phys. 87, 5497 (1987).

${ }^{110}$ M. R. Hand and S. Holloway, Surf. Sci. 211-212, 940 (1989).

${ }^{111}$ G. R. Darling and S. Holloway, Rep. Prog. Phys. 58, 1595 (1995).

${ }^{112}$ A. Gross and M. Scheffler, Phys. Rev. B 57, 2493 (1998).

${ }^{113}$ L. B. F. Juurlink, D. R. Killelea, and A. L. Utz, Prog. Surf. Sci. 84, 69 (2009).

${ }^{114}$ H. A. Michelsen, C. T. Rettner, and D. J. Auerbach, Surf. Sci. 272, 65 (1992). 